\title{
The Twin Physics Interpretation of Gravitational Waves
}

\author{
Anna C.M. Backerra ${ }^{1}$ \\ ${ }^{1}$ Gualtherus Sylvanusstraat 2, 7412 DM Deventer, The Netherlands \\ Correspondence: Anna C.M. Backerra, Gualtherus Sylvanusstraat 2, 7412 DM Deventer, The Netherlands. E-mail: \\ annabackerra@gmail.com \\ Received: January 10, 2018 \\ Accepted: January 25, $2018 \quad$ Online Published: January 30, 2018 \\ doi:10.5539/apr.v10n1p23 \\ URL: https://doi.org/10.5539/apr.v10n1p23
}

\begin{abstract}
Gravitational waves may originate in two approaching black holes, circling around each other until they merge together. This phenomenon is considered by using twin physics, based upon a complementary mathematical language, after fitting Einstein's idea about the relationship between time and space into it. According to the description obtained, the two black holes are annihilated as soon as they touch each other; only after a rearrangement of the constituent Heisenberg-units, these being units of potential energy, one new black hole is generated. During this rearrangement, a pair of oppositely charged particles appears, which is considered to be the birth of electromagnetism. This occurs during a period of time which is principally too short to measure, which seems to be the reason for the apparent merging of the black holes. The expectation is that extraordinarily large signals will be repeatedly received, related to a chain of annihilations of black solid particle pairs and subsequent generations of single black solid particles. We suppose that these signals represent gravitational waves.
\end{abstract}

Keywords: black holes, complementarity, electromagnetism, gravitational waves, twin physics

\section{Introduction}

The first time that gravitational waves are mentioned is in a publication by Poincaré (1905). He supposes that all forces originate in electromagnetism, which was generally accepted at that time, and asks himself how the law of gravitation has to be adapted in order to incorporate this notion. In the discussion he supposes that gravity is not acting instantaneously but with the velocity of light, and that an attracted body is hit by a gravitational wave, sent by the attracting body.

Albert Einstein adopted this concept, combined it with his general theory of relativity and predicted the existence of gravitational waves (1916). He treats gravity as originating in the curvature of spacetime, caused by the presence of mass. As objects with mass move around in spacetime, the curvature changes to reflect their changed locations. If these objects accelerate, the generated changes propagate outward from their source at the speed of light in a wave-like manner and this phenomenon is called 'gravitational waves'. The first observation of gravitational waves was made on 14 September 2015 in which ripples in spacetime were spotted, as announced by the LIGO Scientific Collaboration and Virgo collaborations on 11 February 2016 (Abbott, 2016).

Twin physics does not contain the concept of forces, as a consequence of the introduction of space as an independent, finite item complementary to mass, allowing instantaneous influences. This model is built up completely in accordance with the advises of Heisenberg (1930/1949), Einstein (1996/ original 1936-1950) and Jammer (1974) for the creation of a new theory, combining small- and large-scale phenomena, as well as determinate and indeterminate aspects of nature. It is challenging to describe the origin of gravitational waves in this way, to find out if the suppositions made by Poincaré and Einstein are a necessary starting point for a proper description, or if this phenomenon may also be described by using twin physics.

The basics can be found in five previous publications (Backerra 2010, 2012, 2014, 2016a, 2016b), the last one starting with a manual for the use of twin physics. A more thorough overview of all publications is in preparation for publication by Lambert Academic Publishing in the form of a so-called symposium. For convenience we will repeat the most important principles, concepts and definitions in the next section; elaborated formulas can be found in the appendix. A proper use of twin physics does require some practice. However, after some time it will become a rather easy and quick method, once the principles and concepts are comprehended. 


\section{Theoretical Background of Twin Physics}

The philosophical principle underlying twin physics is the conception of space as an independent, finite entity, having a relatively very low energy density compared with mass. The mathematical principle is the joint description of complementary items by using sets according to Kahn (1967). The physical principle is a renewed conception of energy: Conservation of energy is restricted to conservation of actual energy; potential energy is considered to be a mathematical item which plays no role in this law. Thus mathematical and physical items are kept strictly separated; the first have to be transformed explicitly in order to obtain the second.

The Heisenberg-unit, in short called H-unit and indicated by $H_{i}$, is an elementary unit of potential energy, expressed in complementary mathematical items in three qualities, being time, space and possibly mark (being the precursor of charge and electromagnetism). Each quality is supplied with determinate and indeterminate attributes. Only by interacting with another H-unit, this potential energy can be converted into actual energy.

An interaction is in general written as $H_{i}{ }^{*} H_{j}$.

A set of attributes of $H_{i}$ in general contains four mathematical attributes, being complementary pairwise and written as:

$$
h_{i}=\left\{D_{i}, U^{i}, d_{i}, u^{i}\right\}
$$

in which capitals indicate major determinate and indeterminate attributes and small letters indicate minor determinate and indeterminate attributes. A lower index indicates a determinate attribute, a higher index an indeterminate attribute, so $D_{i}$ is a major determinate attribute, $U^{i}$ a major indeterminate, $d_{i}$ a minor determinate and $u^{i}$ a minor indeterminate one.

The set of time attributes of $H_{i}$ is:

$$
h_{i}(\tilde{t})=\left\{\widetilde{T}_{i}, \widetilde{F}^{i} \backslash \widetilde{T}_{i}, \tilde{\tau}_{i}, \tilde{f}^{i}\right\}
$$

containing a point of time $\widetilde{T}_{i}$, a deliberately large future $\widetilde{F}^{i} \backslash \tilde{T}_{i}$, an infinitesimally small flash of time $\tilde{\tau}_{i}$ and a flying time $\tilde{f}^{i}$ presumably in the order of seconds (see Figure 1). The tildes indicate mathematical items, as a distinction with physical items.

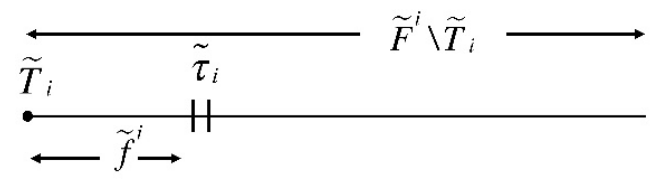

Figure 1. Schematic representation of time attributes of one H-unit

The set of space attributes of $H_{i}$ is:

$$
h_{i}(\tilde{\mathbf{x}})=\left\{\widetilde{P}_{i}, \tilde{S}^{i} \backslash \widetilde{P}_{i}, \tilde{p}_{i}, \tilde{s}^{i}\right\}
$$

containing a point of space $\widetilde{P}_{i}$, a spherical major space $\tilde{S}^{i} \backslash \widetilde{P}_{i}$, a pellicle $\widetilde{p}_{i}$ and a spherical minor space $\tilde{s}^{i}$ (see Figure 2). The minor space has an atomic or molecular size, depending if the H-unit is marked or not. The pellicle is an infinitesimal thin layer upon the minor space, so being a mathematically singular object. 


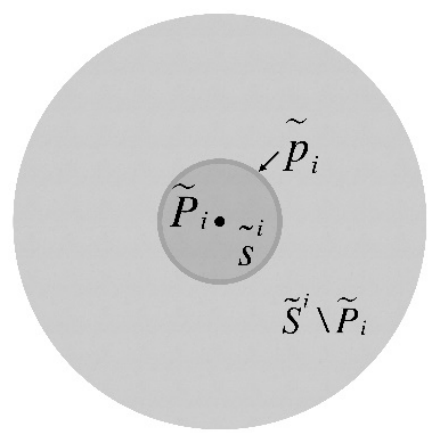

Figure 2. Schematic representation of the space attributes of one H-unit

The set of mark attributes of a marked H-unit is:

$$
h_{i}(\tilde{q})=\left\{\left\{\widetilde{Q}_{i}, \widetilde{\mathbf{E}}_{i}\right\},\left\{\widetilde{Q}_{i} \times i, \widetilde{\mathbf{B}}^{i}\right\},\{1, \widetilde{\nabla}\},\{i, \partial / \partial \tilde{t}\}\right\},
$$

containing an electric set, a magnetic set, a nabla set and a time derivative set.

The letter $i$ on the right of $\widetilde{Q}_{i} \times i$ and in the fourth element represent the unity of imaginary numbers.

If the sign of the charge is relevant, $H_{i}$ may be indicated by $H_{i}^{+}$or $H_{i}^{-}$.

A neutral H-unit may be indicated by $H_{0}$; it has an empty set of mark attributes. Its major and minor spaces are much larger than those of a marked H-unit, because a marked H-unit $H_{i}$ uses a part of its potential energy

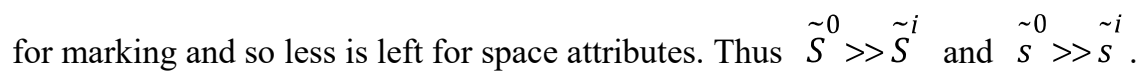

The zipper is a set of four elements, in which all mathematical information about an interaction between $H_{i}$ and $H_{j}$, being two positive or negative marked or neutral H-units, is collected, and transformed into physical time, space and mark. This formula, being a set of four elements, is basically the only one in twin physics; all others are insertions of time, space and mark expressions into this one. When more H-units are involved, they may interact two by two in all possible combinations; the law of conservation of actual energy decides which interactions will become actual. In general the zipper $Z\left(H_{i}^{*} H_{j}\right)=\left\{z_{1}, z_{2}, z_{3}, z_{4}\right\}$ can for each quality of interaction $H_{i}{ }^{*} H_{j}$ be written as a set of four zips in which each zip is a set of two elements:

$$
Z\left(H_{i} * H_{j}\right)=\left\{\begin{array}{l}
\left\{\left[D_{i} \propto D_{j}\right],\left[\left(D_{i} \triangleright u^{i}\right) \propto\left(D_{j} \triangleright u^{j}\right) \propto\left(D_{i} \triangleright u^{j}\right) \propto\left(D_{j} \triangleright u^{i}\right)\right]\right\} \\
\left\{\left[U^{i} \propto U^{j}\right],\left[\left(U^{i} \bowtie d_{i}\right) \propto\left(U^{j} \bowtie d_{j}\right) \propto\left(U^{i} \triangleright d_{j}\right) \propto\left(U^{j} \bowtie d_{i}\right)\right]\right\} \\
\left\{\left[D_{i} \propto U^{j}\right],\left[\left(D_{i} \triangleright u^{i}\right) \propto\left(U^{j} \triangleright d_{j}\right) \propto\left(D_{i} \triangleright d_{j}\right) \propto\left(U^{j} \triangleright u^{i}\right)\right]\right\} \\
\left\{\left[D_{j} \propto U^{i}\right],\left[\left(D_{j} \triangleright u^{j}\right) \propto\left(U^{i} \bowtie d_{i}\right) \propto\left(D_{j} \triangleright d_{i}\right) \propto\left(U^{i} \triangleright u^{j}\right)\right]\right\}
\end{array}\right\} .
$$

In this set, three distinct operators occur, which are defined as follows. The join operator, applied on one major attribute $X$ and one minor attribute $y$, is defined such that these attributes occur combined in the described phenomenon. The link operator $\propto$, applied on the joined pairs $\left(X_{1} \bowtie y_{1}\right)$ and $\left(X_{2} \bowtie y_{2}\right)$, is defined such that, after transformation into a physical space, these pairs occur combined in the described phenomenon. The transformation of mathematical object $\widetilde{X}$ into a physical space, $[X]$, is an operation which changes the mathematical object into a physical item $A$, written as $[X]=A$. In general actual energy is ascribed to it. 
Most transformations are obtained by simply removing the tilde, like $\left[\widetilde{P}_{i}\right]=P_{i}$ or $\left[\widetilde{T}_{i}\right]=T_{i}$, resulting in physical items like a point of space or time. Some less obvious items get a new presentation after having transformed them: For a major space is $\left[\tilde{S}^{i} \backslash \widetilde{P}_{i}\right]=\Theta\left(S^{i} \backslash P_{i}\right)$, called a macrospace; for a minor space is $\left[\begin{array}{c}\tilde{s}^{i} \\ \end{array}\right]=\theta\left(s^{i}\right)$, called a microspace. The pellicle is an exception; its transformation is $\left[\tilde{p}_{i}\right]=\circ\left(p_{i}\right)$ or $\left[\tilde{p}_{i} \cap \tilde{p}_{j}\right]=\circ\left(p_{i} \cap p_{j}\right)$, called a pelletspace; this is a tiny sphere inside two intersecting pellicles, having a diameter equal to the width of a pellicle. For the intersection of a pellicle and a minor space, the transformation is $\left[\tilde{s}^{i} \cap \tilde{p}_{j}\right]=\odot\left(s^{i} \cap p_{j}\right)$, called a dotspace, having the shape of a curved disc. For the flash of time the transformation is $\left[\tilde{\tau}_{i}\right]=d t$, called the time differential and for an intersection of flashes $\left[\tilde{\tau}_{i} \cap \tilde{\tau}_{j}\right]=d t^{2}$, called the second time differential.

One element of the zipper is called a $\boldsymbol{z} \boldsymbol{i} \boldsymbol{p} z_{n}$, with $n \in\{1,2,3,4\}$. This is again a set, but with only two elements, of which the first describes the large- and the second the small-scale physical aspect of a phenomenon. The appearance of a zip is the reconciliation of these two aspects into one expression, written as $A_{i}(t), A_{i}(\mathbf{x})$ and $A_{i}(q)$ for time, space and mark, respectively. A Heisenberg-event (H-event) $\Omega_{n}$ generated by interaction $H_{i}{ }^{*} H_{j}$ is the identification of the time- space- and mark-appearances, described by one zip $z_{n}$, with a physical phenomenon $\varphi_{n}$. This can be written as:

$$
\Omega_{n}\left(H_{i}, H_{j}\right)=\varphi_{n}\left(A_{n}(t), A_{n}(\mathbf{x}), A_{n}(q)\right),
$$

with $n \in\{1,2,3,4\}$, so one interaction may generate more than one $\mathrm{H}$-event. Time is written at the first place, because this appearance is deciding for the other two.

To consider a specific phenomenon, two or more H-units have to be chosen in the proper relative time and space position. In total we distinguish 4 distinct time cases, 7 space cases for equally sized H-units and 12 more for different sized H-units. All cases used in this paper are listed in the Appendix. In general we suppose that in principle each time case may be combined with each space case to find realistic descriptions of phenomena.

In the derivation of twin physics we deviated as little as possible from Einstein's theory and suggestions, and so we treated space and time mathematically in an analogous way (Einstein, 1996/ original 1936-1950). Then the only difference between space and time is in their dimensions, space being three-dimensional and time one-dimensional. This results in an essential asymmetry in the formulations of time and space cases and consequently there is no one-to-one relationship between time and space in twin physics. When using four-dimensional space-time, a part of our possible descriptions would get lost. Time case 1 is for instance analogous to both space cases 1 and 2, and both may describe particles at an atomic scale; this might be one of the reasons that the theory of Einstein about astronomic phenomena is not compatible with quantum mechanics.

On the other hand, we might lose information about the relationship between time and space by the explicit transformation from mathematics to physics and this information might be useful, especially in astronomy which was the subject of Einstein. So to consider gravitational waves, we checked all space and time cases to find out which have analogous definitions, if corrected to the number of dimensions. To get affiliation with the predictions of Einstein about gravitational waves, we will use only these combinations, which are mentioned in the appendix.

Basically there are two types of spatial objects in twin physics: compact and extended spaces. The energy density of a compact space, being a mass-carrying item, is supposed to be one constant value. Consequently, overlapping compact spaces are not allowed. The energy density of an extended space, which is analogous to a classical space, but carrying energy and restricted to a finite area, is minimal the proportion between the potential energy of one neutral $\mathrm{H}$-unit and the volume of a neutral major space; the maximum density obviously cannot be higher than that of a compact space and it might be smaller. Thus extended spaces are allowed to overlap each 
other; then the energy density is an addition of densities up to this unknown maximum; once this is reached, no more extended spaces can be generated inside this area.

In previous publications (Backerra, 2010; 2012) we identified a series of generated items; in this paper only the groups to which relevant particles and macrospaces belong, are used. Two H-units may generate a solid particle $\sigma$ if two minor spaces overlap each other far enough; this can be described by zip $z_{1}$. It may be neutral or charged and identified with a neutron or proton. A pellet particle $\pi$ may be generated if two pellicles are intersecting or coinciding; it is neutral, much smaller than the solid particle, and can be described by zip $z_{2}$. It may be identified with a gluon, a so-called spin particle or a neutrino. A dot particle $\delta$ may be generated if a minor space intersects a pellicle; it may be neutral or charged, has much less mass than the solid particle and can be described by zips $z_{3}$ or $z_{4}$. It may be identified with an electron or positron; then a pellet particle may act as its spin. A point particle $\Pi$ is the lower border of a dot particle, occupying a point of space and so having zero size and no mass; it can store only mark energy. A point particle will only be indicated as $\Pi\left(P_{i}\right)$ if it is the only space item in a zip, otherwise only by point of space $P_{i}$.

With zip $z_{2}$ all macrospaces can be described. A neutral macrospace $\Theta_{0 i 0 j}$ may be generated if two neutral major spaces are intersecting; its size may be astronomic. A magnetic macrospace $\Theta_{i j}^{\mathbf{B}}$ may be generated if two marked major spaces are intersecting; its size is molecular or larger, but not astronomic, and contains magnetic fields or derivations of it. A mixed macrospace $\Theta_{i 0 j}^{\mathbf{B}}$ may be generated if one marked and one neutral major space are interacting; it contains as well magnetic fields or derivations and its size is the same as previous one.

Two marked $\mathrm{H}$-units $H_{i}$ and $H_{j}$ may generate a charged solid particle of type 1, indicated by $\sigma_{i j}^{+}$or $\sigma_{i j}^{-}$. One marked H-unit $H_{i}$ and one neutral H-unit $H_{0 i}$ may generate a charged solid particle of type 2, indicated by $\sigma_{i 0 i}^{+}$or $\sigma_{i 0 i}^{-}$.

Two neutral H-units $H_{0 i}$ and $H_{0 j}$ may generate a black solid particle, indicated by $\sigma_{0 i 0 j}$. These H-units have only non-marked attributes and so this particle cannot store mark energy, thus it carries no charge or electromagnetic field. Because it plays a crucial role in this paper, we will estimate its radius by using a not yet published description of a diatonic hydrogen molecule, in which the proton is considered as a charged solid particle. Then the radius of a black particle is about one quarter of the length of this molecule (being about 0.74 $\AA$ ), so its radius is about 0.18 A. This is an order of $10^{4}$ larger than the order of the radius of a proton (supposed to be about $10^{-5} \AA$ ), so a black solid particle has $10^{12}$ times more mass than a proton (being about $1.67 \times 10^{-27} \mathrm{~kg}$ ) Thus the estimated mass of a black solid particle is in the order of $10^{-15} \mathrm{~kg}$.

In the classical notion, a black hole is supposed to exist in a singularity of spacetime, causing gravity to be so strong that no light can get out and thus the black hole is invisible. This problem is inherited from Poincare (1905), because since the publication of the vector notation of the laws of Maxwell in 1884, these seemed to be the most stable and basic laws in physics and so it was obvious to consider them as basic for all phenomena.

In the twin physics view, on the contrary, gravity cannot be considered as a field. Instead of a singular point in a field, a mathematical singular space object is defined, being the pellicle.

In the next section we will describe the interaction process of two black solid particles, turning around each other. We will indicate what would happen if it were charged solid particles, instead of neutral ones, to show the essential differences; it is possible to compare them because both neutral and charged particles are described by the first zip. This is not the case for charged point particles; they are described by the third or fourth zip and so a similar description of the developing of interactions is not possible.

\section{Two Black Holes of the Smallest Size}

According to Smith (2017), a black hole may have any size, even as small as one atom, so we suppose that two black solid particles can represent two black holes of the smallest size. We will consider them turning around each other, to see if we can understand the occurrence of gravitational waves in twin terms. Four neutral $\mathrm{H}$-units are used, indicated by $H_{01}, H_{02}, H_{03}$ and $H_{04}$, so we have maximum six interactions: $H_{01} * H_{02}, H_{01} * H_{03}$, $H_{01} * H_{04}, H_{02} * H_{03}, H_{02} * H_{04}$ and $H_{03} * H_{04}$. Besides of that, one single marked H-unit $H_{i}$ is considered, as an example for more marked H-units. All together this is called a cluster of H-units. All zippers can be derived by using the appendix.

The zipper for a black solid particle is described in time case 1 and space case 1 , so $\widetilde{T}_{01} \cap \widetilde{T}_{02}=\widetilde{T}_{01}$ and

$\widetilde{P}_{01} \cap \widetilde{P}_{02}=\widetilde{P}_{01}$, and thus $\tilde{S}^{01} \cap \tilde{S}^{02}=\tilde{S}^{01}$. As the first interaction we choose $H_{01} * H_{02}$. Then the zipper is: 


$$
Z\left(H_{01} * H_{02}\right)=\left\{\begin{array}{l}
\left\{\left\{T_{01}, P_{01}, \varnothing\right\},\left\{f^{01}, \theta\left(s^{01}\right), \varnothing\right\}\right\} \\
\left\{\left\{F^{01} \backslash T_{01}, \Theta\left(s^{01} \backslash P_{01}\right), \varnothing\right\},\left\{d t, \circ\left(p_{01}\right), \varnothing\right\}\right\}
\end{array}\right\},
$$

and the set of $\mathrm{H}$-events is:

$$
\Omega\left(H_{01} * H_{02}\right)=\left\{\begin{array}{l}
\sigma_{0102}\left(T_{01} \cup f^{01}, P_{01} \cup \theta\left(s^{01}\right)\right) \\
\pi_{0102}\left(d t, \circ\left(p_{01}\right)\right)
\end{array}\right\},
$$

shortly written as:

$$
\Omega\left(H_{01} * H_{02}\right)=\left\{\sigma_{0102}\left(s^{01}\right), \pi_{0102}\left(p_{01}\right)\right\} .
$$

According to the time appearance $T_{01} \cup f^{01}$, the generated black solid particle $\sigma_{0102}\left(s^{01}\right)$ moves with a constant velocity. It is accompanied by a black pellet particle $\pi_{0102}\left(p_{01}\right)$, which according to the time appearance $d t$, moves with a constant velocity over its surface.

In similar way the second interaction $H_{03} * H_{04}$, generated by $H_{03}$ and $H_{04}$, can be described in the same time and space cases, so $\tilde{S}^{03} \cap \tilde{S}^{04}=\tilde{S}^{03}$. This set of H-events can be written shortly as:

$$
\Omega\left(H_{03} * H_{04}\right)=\left\{\sigma_{0304}\left(s^{03}\right), \pi_{0304}\left(p_{03}\right)\right\},
$$

describing a second black solid particle $\sigma_{0304}\left(s^{03}\right)$ and a second black pellet particle $\pi_{0304}\left(p_{03}\right)$ moving over its surface. The two solid particles $\sigma_{0102}\left(s^{01}\right)$ and $\sigma_{0304}\left(s^{03}\right)$ may move towards or from each other. Note that no macrospace is generated.

The third interaction $H_{01} * H_{03}$ can be described in space case 7 . Space case 7 is characterized by $\tilde{S}^{01} \cap \tilde{S}^{03} \neq \varnothing, \widetilde{P}_{01} \notin \tilde{S}^{03}$ and $\widetilde{P}_{03} \notin \tilde{S}^{01}$, so the points of space of the two black solid particles are outside of the intersections of major spaces. The analogous time case is $3 \boldsymbol{B}$, characterized by $\widetilde{F}^{01} \cap \tilde{\tau}_{03}=\varnothing$.

The zipper has only one element:

$$
Z\left(H_{01} * H_{03}\right)=\left\{\left\{F^{01} \backslash T_{01} \cap F^{03}, \Theta\left(S^{01} \backslash P_{01} \cap S^{03} \backslash P_{03}\right), \varnothing\right\},\{\varnothing, \varnothing, \varnothing\}\right\},
$$

and the $\mathrm{H}$-event is:

$$
\Omega\left(H_{01} * H_{03}\right)=\Theta_{0103}\left(F^{01} \backslash T_{01} \cap F^{03},\left(S^{01} \backslash P_{01} \cap S^{03} \backslash P_{03}\right), \varnothing\right),
$$

so a neutral macrospace $\Theta_{0103}\left(S^{01} \backslash P_{01} \cap S^{03} \backslash P_{03}\right)$ is generated. Equation (12) is in time case 3B, describing cyclic phenomena and thus neutral macrospace $\Theta_{0103}$ is turning around points of space $\widetilde{P}_{01}$ and $\widetilde{P}_{03}$, taking particles $\sigma_{0102}$ and $\sigma_{0304}$ with it. According to equation (8), the particles move towards or away from each other with a constant velocity; we consider the case that they move towards each other. So the two black solid particles are turning around each other and approaching. 
Similar, the fourth, fifth and sixth interactions, being $H_{01} * H_{04}, H_{02} * H_{03}$ and $H_{02} * H_{04}$ are derived.

Using $\tilde{S}^{01} \cap \tilde{S}^{02}=\tilde{S}^{01}$ and $\tilde{S}^{03} \cap \tilde{S}^{04}=\tilde{S}^{03}$, they generate respectively macrospaces $\Theta_{0104}\left(S^{01} \backslash P_{01} \cap S^{03} \backslash P_{03}\right)$, $\Theta_{0203}\left(S^{01} \backslash P_{01} \cap S^{03} \backslash P_{03}\right)$ and $\Theta_{0204}\left(S^{01} \backslash P_{01} \cap S^{03} \backslash P_{03}\right)$.

All occupy the same area as $\Theta_{0103}$ and so its energy density is four times the value for one neutral macrospace.

Because $\widetilde{P}_{01}$ and $\widetilde{P}_{03}$ approach each other, at some moment interaction $H_{01} * H_{03}$ will reach space case 6, in which the points of space enter each other's major spaces, characterized by $\tilde{p}_{01} \cap \tilde{p}_{03}=\varnothing, \widetilde{P}_{01} \in \tilde{S}^{03}$ and $\widetilde{P}_{03} \in \tilde{S}^{01}$. The analogous time case $3 B$ is characterized by $\widetilde{F}^{01} \cap \tilde{\tau}_{03}=\varnothing$. Then the zipper is:

$$
Z\left(H_{01} * H_{03}\right)=\left\{\begin{array}{l}
\left\{\left\{F^{01} \backslash T_{01} \cap F^{03}, \Theta\left(S^{01} \backslash P_{01} \cap S^{03} \backslash P_{03}\right), \varnothing\right\},\{\varnothing, \varnothing, \varnothing\}\right\} \\
\left\{\left\{T_{01}, P_{01}, \varnothing\right\},\{\varnothing, \varnothing, \varnothing\}\right\}
\end{array}\right\} .
$$

In the second element, a point particle $\Pi\left(P_{01}\right)$ is described, but this cannot store space energy, and mark energy is not available, so it cannot appear and the set of H-events contains only one element:

$$
\Omega\left(H_{01} * H_{03}\right)=\Theta_{0103}\left(F^{01} \backslash T_{01} \cap F^{03}, S^{01} \backslash P_{01} \cap S^{03} \backslash P_{03}, \varnothing\right) .
$$

Again only neutral macrospace $\Theta_{0103}\left(S^{01} \backslash P_{01} \cap S^{03} \backslash P_{03}\right)$ is generated. In principle this is the same item as appeared in equation (12), only the area has become larger. But there is a difference: the overlapped points of space are excluded from the major spaces and so we now have a neutral macrospace with two point holes in it.

The fourth, fifth and sixth interactions again describe the same area.

All together we now have two black solid particles $\sigma_{0102}\left(s^{01}\right)$ and $\sigma_{0304}\left(s^{03}\right)$, each with a black pellet particle upon their surface, respectively $\pi_{0102}\left(p_{01}\right)$ and $\pi_{0304}\left(p_{03}\right)$ (see Figure 3). They are turning around each other and approaching, inside the addition of neutral macrospaces $\Theta_{0103}\left(S^{01} \backslash P_{01} \cap S^{03} \backslash P_{03}\right)$, $\Theta_{0104}\left(S^{01} \backslash P_{01} \cap S^{03} \backslash P_{03}\right), \quad \Theta_{0203}\left(S^{01} \backslash P_{01} \cap S^{03} \backslash P_{03}\right)$ and $\Theta_{0204}\left(S^{01} \backslash P_{01} \cap S^{03} \backslash P_{03}\right)$.

For two oppositely charged particles, we would obtain similar results. If they are of type 1, generated respectively by two positive marked H-units and two negative marked H-units, they are indicated by $\sigma_{12}^{+}\left(s^{1}\right)$ and $\sigma_{34}^{-}\left(s^{3}\right)$; they have four identical magnetized spaces around them. If they are of type 2 , generated by a marked and a neutral H-unit, they are indicated by $\sigma_{102}^{+}\left(s^{1}\right)$ and $\sigma_{304}^{-}\left(s^{3}\right)$; then one macrospace is neutral. 


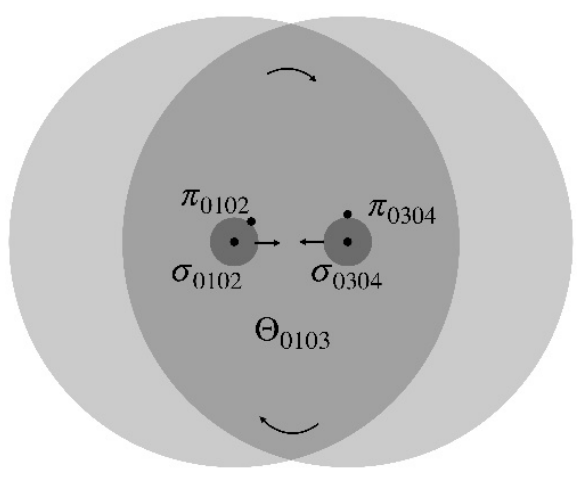

Figure 3. Two black holes in each other's vicinity, inside the addition of four neutral macrospaces, turning around each other and approaching. Only one neutral macrospace is indicated

\section{Charged Point Particles Around the Black Hole}

When in the area of $\left(S^{01} \backslash P_{01} \cap S^{03} \backslash P_{03}\right)$ marked H-units exist, a seventh interaction comes into play; in fact much more of the same type of these interactions may pop up. We take marked H-unit $H_{i}$ as a representative of them and indicate one of these interactions by $H_{i} * H_{01}$. This can be described in space case $7 \boldsymbol{B}$, characterized by $\tilde{p}_{i} \cap \tilde{p}_{01}=\varnothing, \widetilde{P}_{i} \in \tilde{S}^{01}, \widetilde{P}_{01} \notin \tilde{S}^{i}$. The analogous time case is $3 \boldsymbol{B}$, characterized by $\widetilde{F}^{i} \cap \tilde{\tau}_{01}=\varnothing$. Then the zipper is:

$$
Z\left(H_{i}^{*} H_{01}\right)=\left\{\begin{array}{l}
\left.\left\{\left\{F^{i} \backslash T_{i} \cap F^{01}, \Theta\left(S^{i} \backslash P_{i} \cap S^{01} \backslash P_{01}\right),\left[\widetilde{\mathbf{B}}^{i}\right]\right\},\left\{\varnothing, \varnothing,\left[\widetilde{\nabla} \times \widetilde{\mathbf{B}}^{i}\right]\right\}\right\}\right\}, \\
\left\{\left\{T_{i}, P_{i},\left\{Q_{i},\left[\widetilde{\mathbf{E}}_{i}\right]\right\}\right\},\left\{\varnothing, \varnothing,\left[\partial \widetilde{\mathbf{E}}_{i} / \partial \tilde{t} \propto \widetilde{\nabla} \times \widetilde{\mathbf{E}}_{i}\right]\right\}\right\}
\end{array}\right\},
$$

so the set of H-events is:

$$
\Omega\left(H_{i} * H_{01}\right)=\left\{\begin{array}{l}
\Theta_{i 01}\left(F^{i} \backslash T_{i} \cap F^{01}, S^{i} \backslash P_{i} \cap S^{01} \backslash P_{01}, \nabla \times \widetilde{\mathbf{B}^{i}}\right) \\
\Pi_{i 01}\left(T_{i}, P_{i}, Q_{i}\right)
\end{array}\right\} .
$$

The first element describes a magnetized macrospace $\Theta_{i 01}^{\mathbf{B}}\left(S^{i} \backslash P_{i} \cap S^{01} \backslash P_{01}\right)$. It is overlapped by $\Theta_{0103}\left(S^{01} \backslash P_{01} \cap S^{03} \backslash P_{03}\right)$ and so its energy will be added to this neutral macrospace. The second element describes a charged point particle $\Pi_{i 01}\left(P_{i}\right)$, so set of H-events can be written shortly as:

$$
\Omega\left(H_{i}^{*} H_{01}\right)=\left\{\Theta_{i 01}^{\mathbf{B}}\left(S^{i} \backslash P_{i} \cap S^{01} \backslash P_{01}\right), \Pi_{i 01}\left(P_{i}\right)\right\} .
$$

Similar H-events may be generated by the interaction of marked H-units with $H_{02}, H_{03}$ and $H_{04}$.

We suppose that a lot of positive and negative marked H-units are available inside $S^{01} \cap S^{03}$. Because this space has a disc-like shape, the outer areas resemble two wings, swiping over all marked H-units in this area. Charged point particles pop up in the outer areas, and so the tips of these wings produce an electric track as the neutral macrospace is turning around. In the middle area, these point charges can be generated permanently and so we expect a higher and permanent lighting. The artist-impression, as giving in the explanation of black holes by Smith (2017), is a beautiful reflection of this process (see Figure 4). The light part in the middle reflects the area with permanent covered marked H-units. From the tips we see tracks of charged point particles, popping up and disappearing again. 


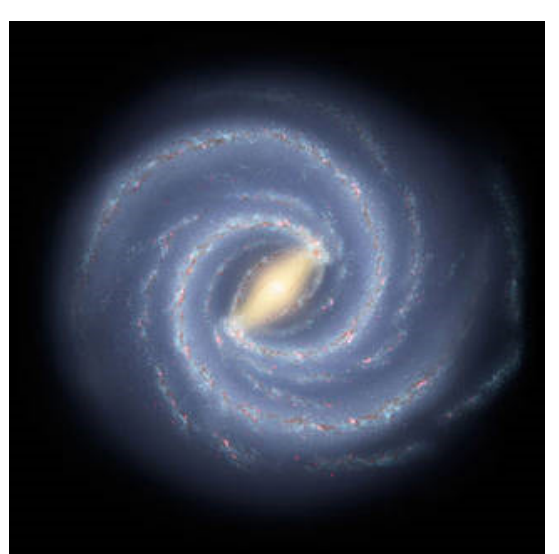

Figure 4. An artist's drawing shows the current view of the Milky Way galaxy. Scientific evidence shows that in the middle of the Milky Way is a supermassive black hole. NASA/JPL-Caltech (NASA 2017)

All together we now have an addition of neutral macrospaces $\Theta_{0103}\left(S^{01} \backslash P_{01} \cap S^{03} \backslash P_{03}\right), \Theta_{0104}\left(S^{01} \cap S^{03}\right)$, $\Theta_{0203}\left(S^{01} \cap S^{03}\right)$ and $\Theta_{0204}\left(S^{01} \cap S^{03}\right)$, with an unknown amount of point particles $\Pi_{i 01}\left(P_{i}\right)$ inside and the same number of mixed macrospaces $\Theta_{i 01}^{\mathbf{B}}\left(S^{i} \backslash P_{i} \cap S^{01} \backslash P_{01}\right)$. Thus the energy density is larger than four times the minimum; moreover, it is not isotropic any more. Inside this area, two black solid particles $\sigma_{0102}\left(s^{01}\right)$ and $\sigma_{0304}\left(s^{03}\right)$ exits, together with their black pellet particles upon their surfaces, respectively $\pi_{0102}\left(p_{01}\right)$ and $\pi_{0304}\left(p_{03}\right)$

\section{Two Black Holes Touching Each Other}

In this section we will consider the touching of the two black solid particles. Interaction $H_{01} * H_{03}$ will change its character as soon as their neutral pellicles touch each other. Then they reach space case 5, characterized by $\tilde{p}_{01} \cap \tilde{p}_{03}=\tilde{p}_{0103} ;$ the analogous time case 2 is characterized by $\widetilde{T}_{01} \cap \widetilde{T}_{03}=\varnothing$ and $\tilde{\tau}_{01} \cap \tilde{\tau}_{03} \neq \varnothing$. The zipper is:

$$
Z\left(H_{01} * H_{03}\right)=\left\{\begin{array}{l}
\left\{\left\{F^{01} \backslash T_{01} \cap F^{03}, \Theta\left(S^{01} \backslash P_{01} \cap S^{03} \backslash P_{03}\right), \varnothing\right\},\left\{d t^{2}, \circ\left(p_{0103}\right), \varnothing\right\}\right\} \\
\left\{\left\{T_{01}, P_{01}, \varnothing\right\},\{\varnothing, \varnothing, \varnothing\}\right\}
\end{array}\right\},
$$

and the set of H-events is:

$$
\Omega\left(H_{01} * H_{03}\right)=\pi_{0103}\left(d t^{2}, \circ\left(p_{0103}\right), \varnothing\right) .
$$

This is a pellet particle $\pi_{0103}\left(p_{0103}\right)$ in the touching point. It is accelerating, but it depends on the continuation of the relative movement of the two $\mathrm{H}$-units how this is possible. So how can $H_{01}$ and $H_{03}$ continue their interaction? They cannot come more close to each other, because two other neutral H-units, being $H_{02}$ and $H_{04}$, are generating two black solid particles in that area and a doubling of (a part of) these compact spaces is not allowed. Thus merging is impossible and so interaction $H_{01} * H_{03}$ has come into a crisis. Nature has only a split-second time to create a solution and all solutions are allowed, if only the total actual energy is conserved and each H-unit stores a constant amount of potential energy.

The only possible escape is a shrinking of two of the four neutral H-units by producing an own identity for each of the H-units $H_{01}$ and $H_{03}$, so by a conversion from neutral H-units into marked H-units. Then the minor spaces will shrink drastically, their pellicles are not touching anymore and the crisis will be solved (see Figure 5). 
Thus a conversion of space energy into mark energy occurs. This is an allowed change, because $H_{01}$ and $H_{03}$ can provide the potential energy, necessary to produce an identity, by spending a part of their space energy to create oppositely mark attributes. Obviously these marks have to be opposite, as otherwise no identity would come into existence. Note that the sudden change of mass, occurring when each of the two black solid particles converts into a marked solid particle, will be described only after the flying time, so the energetic details have to be solved only after this period. We will indicate this change of attributes by:

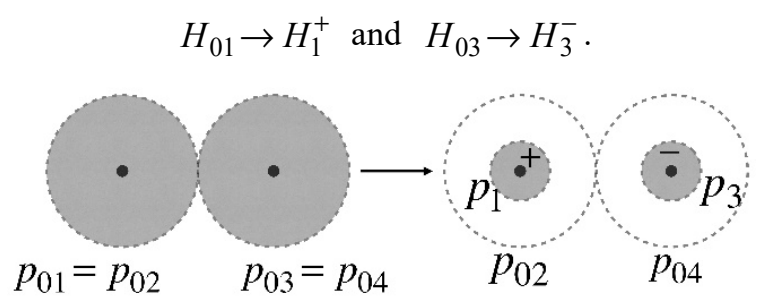

Clearly this solution is impossible for charged particles of type 1, being generated by marked H-units, having already small pellicles and thus no possibility to shrink. In principle it could be a solution that one particle started to turn around the other, to supply a track to the pellet particle, but the time label tells that this had to be accelerated, which makes it energetically impossible. If particles of type 1 reach this space case, they will be annihilated. On the contrary, charged particles of type 2 reach this without any problem, so from now on their story will be parallel to that of the black solid particles.

When $H_{01}$ and $H_{03}$ convert into $H_{1}^{+}$and $H_{3}^{-}$, then interactions $H_{01} * H_{02}$ and $H_{03} * H_{04}$ are converted into $H_{1}^{+*} H_{02}$ and $H_{3}^{-*} H_{04}$. Each of them generates a solid particle, described in time case 1 , characterized by $\widetilde{T}_{1} \cap \widetilde{T}_{02}=\widetilde{T}_{1}$, and space case $9 \boldsymbol{D}$, characterized by $\widetilde{P}_{1} \cap \widetilde{P}_{02}=\widetilde{P}_{1}$. Then the zipper for $H_{1}^{+*} H_{02}$ is:

$$
Z\left(H_{1}^{+} * H_{02}\right)=\left\{z_{1}, z_{2}\right\}=\left\{\begin{array}{l}
\left\{\left\{T_{1}, P_{1}, Q_{1}\right\},\left\{f^{1}, \theta\left(s^{1}\right), \partial \widehat{\mathbf{E}}_{1} / \partial t\right\}\right\} \\
\left\{\left\{F^{1} \backslash T_{1}, \Theta\left(S^{1} \backslash P_{1}\right), \widehat{\mathbf{B}}^{1}\right\},\{d t, \varnothing, \mathbf{O}\}\right\}
\end{array}\right\},
$$

and the set of H-events is:

$$
\Omega\left(H_{1}^{+} * H_{02}\right)=\left\{\begin{array}{l}
\sigma_{102}^{+}\left(T_{1} \cup f^{1}, P_{1} \cup \theta_{1}\left(s^{1}\right),\left\{Q_{1}, \partial \widehat{\mathbf{E}}_{1} / \partial t\right\}\right) \\
\Theta_{102}\left(d t, S^{1} \backslash P_{1}, \widehat{\mathbf{B}}^{1}\right)
\end{array}\right\},
$$

shortly written as:

$$
\Omega\left(H_{1}^{+*} H_{02}\right)=\left\{\sigma_{102}^{+}\left(s^{1}\right), \Theta_{102}^{\mathbf{B}}\left(S^{1} \backslash P_{1}\right)\right\}
$$

The first element describes a charged solid particle of type 2 , indicated by $\sigma_{102}^{+}\left(s^{1}\right)$, in point of space $P_{1}$.

The second element describes a mixed macrospace $\Theta_{102}^{\mathbf{B}}\left(S^{1} \backslash P_{1}\right)$.

Similar for $H_{3}^{-*} H_{04}$ the set of $\mathrm{H}$-events is:

$$
\Omega\left(H_{3}^{-*} H_{04}\right)=\left\{\sigma_{304}^{-}\left(s^{3}\right), \Theta_{304}^{\mathbf{B}}\left(S^{3} \backslash P_{3}\right)\right\} .
$$


The first element describes an oppositely charged solid particle of type 2 , indicated by $\sigma_{304}^{-}\left(s^{3}\right)$, in point of space $P_{3}$; the second element describes a second mixed macrospace $\Theta_{304}^{\mathbf{B}}\left(S^{3} \backslash P_{3}\right)$.

After the conversion, marked $\mathrm{H}$-units $H_{1}^{+}$and $H_{3}^{-}$are not touching anymore; they have only overlapping major spaces. So interaction $H_{1}^{+*} H_{3}^{-}$can be described by space case 6 , characterized by $\widetilde{F}^{1} \cap \tilde{\tau}_{3}=\varnothing$; $\tilde{p}_{1} \cap \tilde{p}_{3}=\varnothing, \quad \widetilde{P}_{1} \in \tilde{S}^{3}$ and $\widetilde{P}_{3} \in \tilde{S}^{1}$; the analogous time case is $3 \boldsymbol{B}$, characterized by $\tilde{\tau}_{1} \cap \tilde{\tau}_{3}=\varnothing$ and $\widetilde{F}^{1} \cap \tilde{\tau}_{3}=\varnothing$. Then the zipper $\left\{z_{2}, z_{3}\right\}$ is:

$$
Z\left(H_{1}^{+} * H_{3}^{-}\right)=\left\{\begin{array}{l}
\left\{\left\{F^{1} \backslash T_{1} \cap F^{3}, \Theta\left(S^{1} \backslash P_{1} \cap S^{3} \backslash P_{3}\right),\left[\widetilde{\mathbf{B}}^{1} \propto \widetilde{\mathbf{B}}^{3}\right]\right\},\left\{\varnothing, \varnothing,\left[\widetilde{\nabla} \times \widetilde{\mathbf{B}}^{1} \propto \widetilde{\nabla} \times \widetilde{\mathbf{B}}^{3}\right]\right\}\right\} \\
\left.\left\{\left\{T_{1}, P_{1},\left\{\left[\widetilde{Q}_{1}\right],\left[\widetilde{\mathbf{E}}_{1} \propto \widetilde{\mathbf{B}}^{3}\right]\right\}\right\},\left\{\varnothing, \varnothing,\left[\partial \widetilde{\mathbf{E}}_{1} / \partial \tilde{t} \propto \widetilde{\nabla} \times \widetilde{\mathbf{B}}^{3} \propto \widetilde{\nabla} \times \widetilde{\mathbf{E}}_{1} \propto \partial \widetilde{\mathbf{B}}^{3} / \partial \tilde{t}\right]\right\}\right\}\right\} .
\end{array}\right\}
$$

Zip $z_{3}$ cannot appear, as $P_{1}$ is occupied by a solid particle, so the set of $\mathrm{H}$-events contains only one element:

$$
\Omega\left(H_{1}^{+} * H_{3}^{-}\right)=\Theta_{13}^{\mathbf{B}}\left(S^{1} \backslash P_{1} \cap S^{3} \backslash P_{3}\right),
$$

which is magnetic macrospace $\Theta_{13}^{\mathbf{B}}\left(S^{1} \backslash P_{1} \cap S^{3} \backslash P_{3}\right)$.

Thus, by the conversion of two neutral H-units into two marked H-units, the black solid particles shrink into charged solid particles, and a magnetic macrospace is generated. We consider this as the birth of electromagnetism.

Next we will consider interaction $H_{02} * H_{04}$, which in the previous steps generated a neutral macrospace. As soon as they touch each other, space case 5 is valid, characterized by $\tilde{p}_{02} \cap \tilde{p}_{04}=\tilde{p}_{0204}$. The analogous time case 2 is characterized by $\widetilde{T}_{02} \cap \widetilde{T}_{04}=\varnothing$ and $\tilde{\tau}_{02} \cap \tilde{\tau}_{04} \neq \varnothing$. Then the zipper is:

$$
Z\left(H_{02} * H_{04}\right)=\left\{\begin{array}{l}
\left\{\left\{F^{02} \backslash T_{02} \cap F^{04}, \Theta\left(S^{02} \backslash P_{02} \cap S^{04} \backslash P_{04}\right), \varnothing\right\},\left\{d t^{2}, \circ\left(p_{0204}\right), \varnothing\right\}\right\} \\
\left\{\left\{T_{02}, P_{02}, \varnothing\right\},\{\varnothing, \varnothing, \varnothing\}\right\}
\end{array}\right\},
$$

and the H-event is:

$$
\Omega\left(H_{02} * H_{04}\right)=\pi_{0204}\left(d t^{2}, \circ\left(p_{0204}\right), \varnothing\right) .
$$

This pellet particle seems to replace the previous one $\pi_{0103}\left(p_{0103}\right)$, which was generated by $H_{01} * H_{03}$ and could not continue to exist after these two H-units converted into $H_{1}^{+}$and $H_{3}^{-}$. The difference is, that this one is free from any solid particle.

The two remaining interactions to consider are $H_{1} * H_{04}$ and $H_{3} * H_{02}$. Their pellicles are not intersecting, which can described in space case $7 \mathrm{C}$ and analogous time case $3 \boldsymbol{B}$. For interaction $H_{1} * H_{04}$, this is characterized by $\tilde{p}_{1} \cap \tilde{p}_{04}=\varnothing, \quad \widetilde{P}_{1} \in \tilde{S}^{04}$ and $\widetilde{P}_{04} \in \tilde{S}^{1} ; \widetilde{F}^{1} \cap \tilde{\tau}_{04}=\varnothing$. Then the zipper is: 


$$
Z\left(H_{1}^{+} * H_{04}\right)=\left\{\begin{array}{l}
\left\{\left\{F^{1} \backslash T_{1} \cap F^{3}, \Theta\left(S^{1} \backslash P_{1} \cap S^{04} \backslash P_{04}\right),\left[\widetilde{\mathbf{B}}^{1}\right]\right\},\left\{\varnothing, \varnothing,\left[\widetilde{\nabla} \times \widetilde{\mathbf{B}}^{1}\right]\right\}\right\} \\
\left\{\left\{T_{1}, P_{1},\left\{\left[\widetilde{Q}_{1}\right],\left[\widetilde{\mathbf{E}}_{1}\right]\right\}\right\},\left\{\varnothing, \varnothing,\left[\partial \widetilde{\mathbf{E}}_{1} / \partial \tilde{t} \propto \widetilde{\nabla} \times \widetilde{\mathbf{E}}_{1}\right]\right\}\right\}
\end{array}\right\},
$$

and the set of H-events is:

$$
\Omega\left(H_{1} * H_{04}\right)=\left\{\begin{array}{l}
\Theta_{104}\left(F^{1} \backslash T_{1} \cap F^{04}, S^{1} \backslash P_{1} \cap S^{04} \backslash P_{04}, \nabla \times \widehat{\mathbf{B}}^{-1}\right) \\
\Pi_{104}\left(T_{1}, P_{1}, Q_{1}\right)
\end{array}\right\} .
$$

The charged point particle $\Pi_{104}\left(Q_{1}\right)$ is not allowed to appear, because in the same point the charge of $\sigma_{102}^{+}$is localized, so only mixed macrospace $\Theta_{104}^{\mathbf{B}}\left(S^{1} \backslash P_{1} \cap S^{04} \backslash P_{04}\right)$ appears. Similar, interaction $H_{3} * H_{02}$ generates a second mixed macrospace $\Theta_{302}^{\mathbf{B}}\left(S^{3} \backslash P_{3} \cap S^{02} \backslash P_{02}\right)$.

All together, as a consequence of the conversion of neutral H-units $H_{01}$ and $H_{03}$ in marked ones $H_{1}^{+}$and $H_{3}^{-}$, two black solid particles with their black pellet particles are annihilated. Also the neutral macrospace is annihilated, together with an unknown number of charged point particles and magnetic macrospaces. During the conversion, pellet particle $\pi_{0103}\left(p_{0103}\right)$ appears, but it is annihilated during the conversion.

Instead, oppositely charged solid particles $\sigma_{102}^{+}\left(s^{1}\right)$ in $P_{1}$ and $\sigma_{304}^{-}\left(s^{3}\right)$ in $P_{3}$ are generated, and pellet particle $\pi_{0204}\left(p_{0204}\right)$ in the touching point of the two remaining neutral H-units (see Figure 6). Moreover, magnetic macrospace $\Theta_{13}^{\mathbf{B}}\left(S^{1} \backslash P_{1} \cap S^{3} \backslash P_{3}\right)$, mixed macrospaces $\quad \Theta_{104}^{\mathbf{B}}\left(S^{1} \backslash P_{1} \cap S^{04} \backslash P_{04}\right)$ and $\Theta_{302}^{\mathbf{B}}\left(S^{3} \backslash P_{3} \cap S^{02} \backslash P_{02}\right)$ are generated (see Figure 7). Note that these three, partly overlapping macrospaces occupy a much smaller region than the previously generated neutral macrospaces.

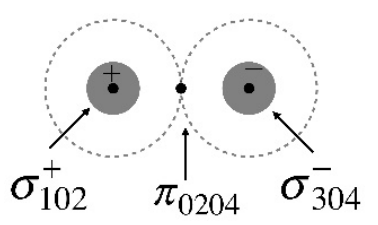

Figure 6. Two charged solid particles and a pellet particle in the touching point.

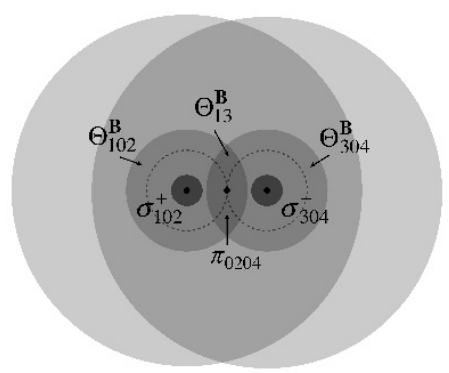

Figure 7. The same situation as above; major spaces and macrospaces also are shown 


\section{Rearrangement of Interactions}

The two opposite charged particles attract each other (see Backerra 2016b) and thus the two involved neutral H-units $H_{02}$ and $H_{04}$ still are approaching. Consequently, the touching point will break open to a pellicle track and so interaction $H_{02} * H_{04}$ enters space case 4, characterized by $\tilde{s}^{02} \cap \tilde{s}^{04} \neq \varnothing, \widetilde{P}_{02} \notin \tilde{s}^{02} \cap \tilde{s}^{04}$ and

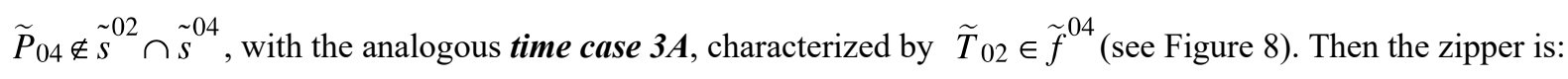

$$
Z\left(H_{02} * H_{04}\right)=\left\{\begin{array}{l}
\left\{\left\{F^{02} \backslash T_{02} \cap F^{04}, \Theta\left(S^{01} \backslash P_{02} \cap S^{04} \backslash P_{04}\right), \varnothing\right\},\left\{\varnothing, \circ\left(p_{02} \cap p_{04}\right), \varnothing\right\}\right\} \\
\left\{\left\{T_{02}, P_{02}, \varnothing\right\},\{\varnothing, \varnothing, \varnothing\}\right\}
\end{array}\right\},
$$

and the $\mathrm{H}$-event is:

$$
\Omega\left(H_{02} * H_{04}\right)=\pi_{0204}\left(F^{02} \backslash T_{02} \cap F^{04}, \circ\left(p_{02} \cap p_{04}\right), \varnothing\right),
$$

which is pellet particle $\pi_{0204}\left(p_{02} \cap p_{04}\right)$. This is the same item as in equation (28), only now traveling with increasing radius through pelletspace $\circ\left(p_{02} \cap p_{04}\right)$. In time case 3A, the time label $F^{02} \backslash T_{02} \cap F^{04}$ overlaps the flash of time $d t^{2}$ and so the neutrino can continue to accelerate. Its actual energy cannot exceed the available potential energy of $H_{01}$ and $H_{03}$. If this limit is reached, the pellet particle can only continue with a constant velocity and so only if time case 1 is possible; if this is not possible because of the other interactions, it will be annihilated.

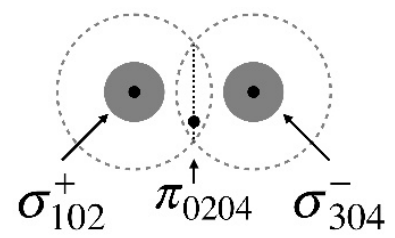

Figure 8 . The charged solid particles are more close; the pellet particle turns around in the plane in between

The approaching of the charged particles continues until both neutral pellicles meet the much smaller marked pellicles. Then the cross-over interactions $H_{1}^{+*} H_{04}$ and $H_{3}^{-} * H_{02}$ might generate two more pellet particles, but this is not allowed, because pellicles $\tilde{p}_{02}$ and $\tilde{p}_{04}$ together already are generating a pellet particle and so $H_{02}$ and $H_{04}$ are not available. Thus each neutral pellicle passes the marked pellicle and $\pi_{0204}\left(p_{02} \cap p_{04}\right)$ continues to travel around.

There will be a moment that point of space $\widetilde{P}_{1}$ enters neutral pellicle $\tilde{s}^{04}$, and point of space $\widetilde{P}_{3}$ enters neutral pellicle $\tilde{s}^{02}$. Then interactions $H_{1}^{+*} H_{04}$ and $H_{3}^{-*} H_{02}$ reach space case $8 \mathrm{C}$, characterized by $\widetilde{P}_{1} \in \widetilde{p}_{04}$ and $\widetilde{P}_{3} \in \widetilde{p}_{02}$, respectively (see Figure 9). 


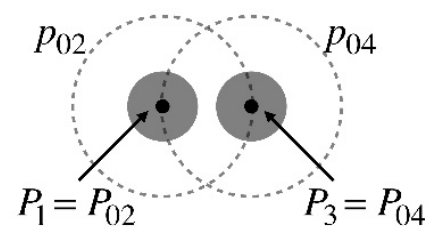

Figure 9 . The points of space reach the neutral pellicles

Now interactions $H_{1}^{+*} H_{02}$ and $H_{3}^{-} * H_{04}$ come into a crisis; if none of them changes, then soon the same problem will arise as we saw in the beginning of this section, because overlapping of solid particles is not allowed. But there is a solution: H-unit $H_{1}^{+}$is allowed to step over from interaction $H_{1}^{+} * H_{02}$ to $H_{1}^{+*} H_{04}$ as its main interaction, generating a charged dot particle with $H_{04}$ instead of a charged solid particle with $H_{02}$. So charged solid particle $\sigma_{102}^{-}\left(s^{1}\right)$ is annihilated and the new interaction $H_{1}^{+*} H_{04}$ starts in space case $8 C$, characterized by $\widetilde{P}_{1} \in \tilde{p}_{04}$, with analogous time case 4 , characterized by $\widetilde{T}_{1} \in \tilde{\tau}_{04}$. Then the zipper is:

$$
Z\left(H_{1}^{+*} H_{04}\right)=\left\{\begin{array}{l}
\left\{F^{1} \backslash T_{1} \cap F^{04}, \Theta\left(S^{1} \backslash P_{1} \cap S^{04} \backslash P_{04}\right),\left[\widetilde{\mathbf{B}}^{1}\right]\right\},\left\{\varnothing, \circ\left(p_{1} \cap p_{04}\right),\left[\widetilde{\nabla} \times \widetilde{\mathbf{B}}^{1}\right]\right\} \\
\left\{\left\{T_{1}, P_{1}, Q_{1}\right\},\left\{f^{1} \cap d t, \odot\left(s^{1} \cap p_{04}\right),\left[\partial \widetilde{\mathbf{E}}_{1} / \partial \tilde{t} \propto \widetilde{\nabla} \times \widetilde{\mathbf{E}}_{1}\right]\right\}\right\}
\end{array}\right\},
$$

so the set of H-events is:

$$
\Omega\left(H_{1}^{+} * H_{04}\right)=\left\{\begin{array}{l}
\pi_{104}\left(F^{1} \backslash T_{1} \cap F^{04}, \circ\left(p_{1} \cap p_{04}\right), \nabla \times \widehat{\mathbf{B}}^{1}\right) \\
\delta_{104}^{+}\left(T_{1} \cup\left(f^{1} \cap d t\right), P_{1} \cup \odot\left(s^{1} \cap p_{04}\right),\left\{Q_{1}, \partial \widehat{\mathbf{E}}_{1} / \partial t+\nabla \times \widehat{\mathbf{E}}_{1}\right\}\right)
\end{array}\right\} .
$$

The first element is a pellet particle $\pi_{104}\left(p_{1} \cap p_{04}\right)$, turning around inside the pellicle with a relatively small radius. The second element is charged dot particle $\delta_{104}^{+}\left(s^{1} \cap p_{04}\right)$, localized in point $P_{1}$, having mass and moving with a constant velocity through the neutral pellicle (see Figure 10). The pellet particle $\pi_{104}$ is close to $\delta_{104}^{+}$, turning around it and so acting as the spin for the dot particle (the notion of spin in twin physics has to be published yet).

Similar, $H_{3}^{-}$steps over from interaction $H_{3}^{-} * H_{04}$ to $H_{3}^{-*} H_{02}$ as its main interaction. Then charged solid particle $\sigma_{304}^{-}\left(s^{3}\right)$ is annihilated. A second charged dot particle $\delta_{302}^{-}\left(s^{3} \cap p_{02}\right)$ is generated in point $P_{3}$, and a second pellet particle $\pi_{302}\left(p_{3} \cap p_{02}\right)$, acting as the spin for the dot particle. 


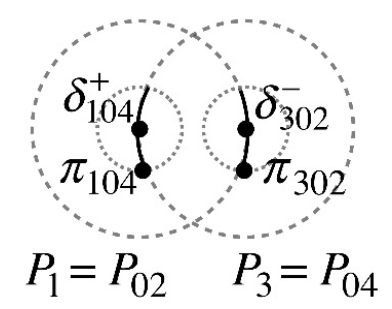

Figure 10. Rearrangement of interactions

During this rearrangement of interactions, the points of space of $H_{02}$ and $H_{04}$ are inside each other's pellicles, so they interact in space case 3, characterized by $\widetilde{P}_{02} \in \widetilde{p}_{04}$ and $\widetilde{P}_{04} \in \widetilde{p}_{02}$, in the analogous time case 4, characterized by $\widetilde{T}_{02} \in \tilde{\tau}_{04}$. In the previous step, pellet particle $\pi_{0204}\left(p_{02} \cap p_{04}\right)$ was generated by them, as described in equation (32). In this time and space case, the same pellet particle is described. Also a second $\mathrm{H}$-event is described, but this is a dot particle which cannot appear because in $P_{02}$ already a dot particle is generated. So the set of H-events does not change and this pellet particle continues to turn around in the same plane (see Figure 11).

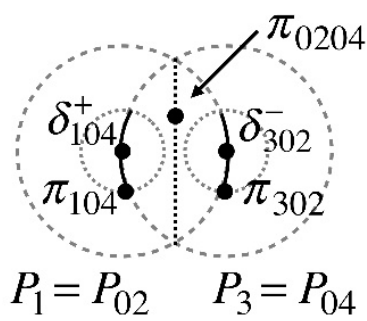

Figure 11. A pellet particle travels through the neutral pellicle intersection in the plane between the charged point particles

We will consider subsequently how the remaining interactions $H_{1}^{+*} H_{3}, H_{1}^{+*} H_{02}$ and $H_{3}^{-*} H_{04}$ will develop in the time that the two solid particles and two pellet particles pop up, and one pellet particle continues to travel around.

- Interaction $H_{1}^{+*} H_{3}$ is not affected by this change and so magnetic macrospac $\Theta_{13}^{\mathbf{B}}\left(S^{1} \backslash P_{1} \cap S^{3} \backslash P_{3}\right)$ still is generated.

- Interaction $H_{1}^{+*} H_{02}$ cannot generate a solid particle anymore, because point of space $P_{1}$ is occupied by the dot particle. Thus the set of H-events (23) is reduced to only the second element, which is mixed macrospace $\Theta_{102}^{\mathbf{B}}\left(S^{1} \backslash P_{1}\right)$.

- Similar, interaction $H_{3}^{-} * H_{04}$ is reduced to mixed macrospace $\Theta_{304}^{\mathbf{B}}\left(S^{3} \backslash P_{3}\right)$.

So by this second conversion, the oppositely charged solid particles transform into two oppositely charged dot particles and their pellet particles. The space energy of the solid particles can only partly be transformed into these much smaller four particles; however, the decrease of actual space energy is not yet violating the energy law, because the annihilation of the two charged solid particles can be described only after the flying time $f^{1}$ in equation (22). During this period, the remaining interactions have the possibility to rearrange such that more space energy is generated. 
All together, we now have charged dot particle $\delta_{104}^{+}\left(s^{1} \cap p_{04}\right)$ in $P_{1}$ with pellet particle $\pi_{104}\left(p_{1} \cap p_{04}\right)$, charged dot particle $\delta_{302}^{-}\left(s^{3} \cap p_{02}\right)$ in $P_{3}$ with pellet particle $\pi_{302}\left(p_{3} \cap p_{02}\right)$, and pellet particle $\pi_{0204}\left(p_{02} \cap p_{04}\right)$ in a plane between them. Moreover, we still have magnetic macrospace $\Theta_{13}^{\mathbf{B}}\left(S^{1} \backslash P_{1} \cap S^{3} \backslash P_{3}\right)$ and the mixed macrospaces $\Theta_{102}^{\mathbf{B}}\left(S^{1} \backslash P_{1}\right)$ and $\Theta_{304}^{\mathbf{B}}\left(S^{3} \backslash P_{3}\right)$ (see Figure 12).

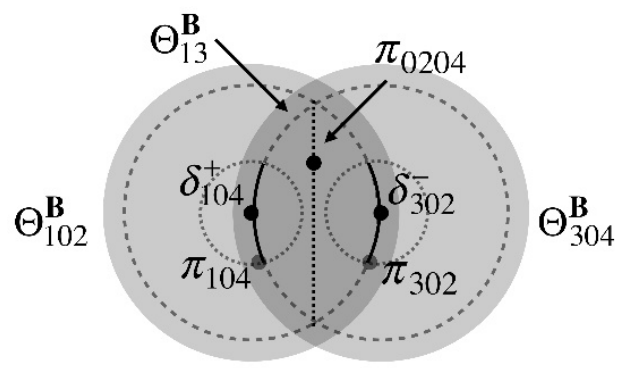

Figure 12. Situation after the rearrangement of interactions

\section{The Generation of One New Black Hole}

As soon as $H_{02} * H_{04}$ leaves space case 3, because $H_{02}$ and $H_{04}$ continue to approach each other, charged points $P_{1}$ and $P_{3}$ (in the middle of the two dot particles), do not join respectively points $P_{02}$ and $P_{04}$ anymore, but stay in the pellicles of $H_{02}$ and $H_{04}$, like being dragged away. So now the four points of space, which were two by two coinciding, shift away from each other (see Figure 13).

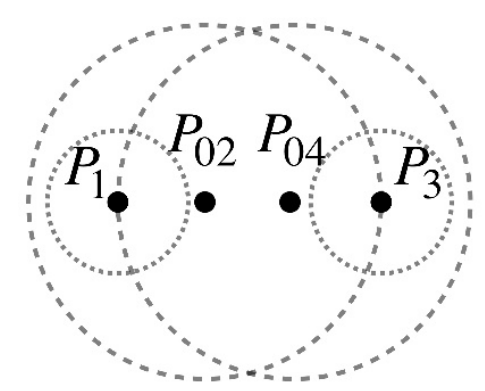

Figure 13. The sequence of the four points of space

The neutral points $\widetilde{P}_{02}$ and $\widetilde{P}_{02}$ will enter the overlapping major space $S^{01} \backslash P_{02} \cap S^{04} \backslash P_{04}$, described in space case 2, characterized by $\widetilde{P}_{02} \cap \widetilde{P}_{04}=\varnothing, \widetilde{P}_{02} \in \tilde{S}^{04}$ and $\widetilde{P}_{04} \in \tilde{S}^{02}$, with analogous time case 1, characterized by $\widetilde{T}_{02} \cap \widetilde{T}_{04}=\widetilde{T}_{02}$. Then the zipper is:

$$
Z\left(H_{02} * H_{04}\right)=\left\{\begin{array}{l}
\left\{\left\{T_{02}, \varnothing, \varnothing\right\},\left\{f^{02}, \theta\left(s^{02} \cap s^{04}\right), \varnothing\right\}\right\} \\
\left\{\left\{F^{02} \backslash T_{02}, \Theta\left(S^{02} \backslash P_{02}\right), \varnothing\right\},\left\{d t, \circ\left(p_{02} \cap p_{04}\right), \varnothing\right\}\right\}
\end{array}\right\} .
$$


and the set of H-events is:

$$
\Omega\left(H_{02} * H_{04}\right)=\left\{\begin{array}{l}
\sigma_{0204}\left(T_{02} \cup f^{02}, \theta\left(s^{02} \cap s^{04}\right), \varnothing\right) \\
\pi_{0204}\left(d t, \circ\left(p_{02} \cap p_{04}\right), \varnothing\right)
\end{array}\right\},
$$

The first element describes a black solid particle $\sigma_{0204}\left(s^{02} \cap s^{04}\right)$; the time label tells that it moves with a constant velocity. In the beginning its volume is about $1 / 3$ of the black solid particle of equation (8), so its mass is about $1 / 3$ of a full-size black solid particle. It might seem as if the original two black holes are merged into this one, but the cluster of H-units has been reconstructed drastically before reaching this. The second element of equation (36) describes a black pellet particle $\pi_{0204}\left(p_{02} \cap p_{04}\right)$ traveling over the surface of the black solid particle (see Figure 14).

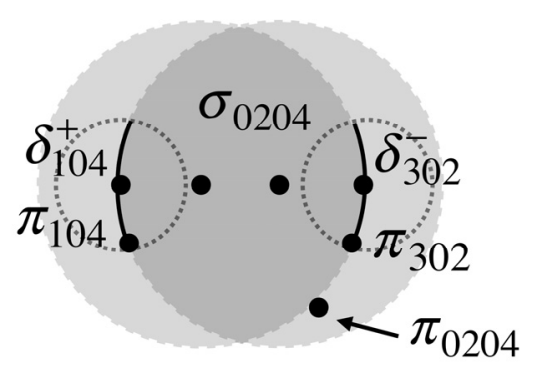

Figure 14. A new black solid particle with a black pellet particle, two charged dot particles and their pellet particles. Major spaces are not shown

The energy balance of the cluster is decided by both the extended (spatial) and the compact (mass-carrying) spaces. If this is not fitting completely, the two H-units will move closer to each other, as their interaction generates more mass if neutral microspace $s^{02} \cap s^{04}$ grows. This goes on until the energy balance is restored, with a maximum when the minor spaces coincide. If indeed the energy balance is reached in the coinciding case for the black solid particle, then we have space case 1, characterized by $\tilde{s}^{02} \cap \tilde{s}^{04}=\tilde{s}^{02}$, and time case 1, characterized by $\widetilde{T}_{02} \cap \widetilde{T}_{04}=\widetilde{T}_{02}$. The zipper is:

$$
Z\left(H_{02} * H_{04}\right)=\left\{\begin{array}{l}
\left\{\left\{T_{02}, \varnothing, \varnothing\right\},\left\{f^{02}, \theta\left(s^{02}\right), \varnothing\right\}\right\} \\
\left\{\left\{F^{02} \backslash T_{02}, \Theta\left(S^{02} \backslash P_{02}\right), \varnothing\right\},\left\{d t, \circ\left(p_{02}\right), \varnothing\right\}\right\}
\end{array}\right\},
$$

and the set of $\mathrm{H}$-events of interaction $\mathrm{H}_{02} * \mathrm{H}_{04}$ finally is:

$$
\Omega\left(H_{02} * H_{04}\right)=\left\{\begin{array}{l}
\sigma_{0204}\left(T_{02} \cup f^{02}, \theta\left(s^{02}\right), \varnothing\right) \\
\pi_{0204}\left(d t, \circ\left(p_{02}\right), \varnothing\right)
\end{array}\right\},
$$

describing black solid particle $\sigma_{0204}\left(s^{02}\right)$ and black pellet particle $\pi_{0204}\left(p_{0204}\right)$ upon its surface. 
We will check the remaining interactions for the coinciding case of $H_{02} * H_{04}$ :

- For interactions $H_{1}^{+} * H_{04}$ and $H_{3}^{-} * H_{02}$ nothing changes, so they continue to generate charged dot particle $\delta_{104}^{+}\left(s^{1} \cap p_{04}\right)$ in $P_{1}$ with pellet particle $\pi_{104}\left(p_{3} \cap p_{04}\right)$, and charged dot particle $\delta_{302}^{-}\left(s^{3} \cap p_{02}\right)$ in $P_{3}$ with pellet particle $\pi_{302}\left(p_{3} \cap p_{02}\right)$. The dot particles exist opposite to each other at the surface of the black solid particle.

- Interaction $H_{1}^{+*} H_{3}^{-}$is in space case 6 and analogous time case 3B. The zipper is equal to equation (25), describing a magnetic macrospace and a point particle; the point particle cannot appear, because both points $P_{1}$ and $P_{3}$ are already occupied by the dot particles. Magnetic macrospace $\Theta_{13}^{\mathbf{B}}\left(S^{1} \backslash P_{1} \cap S^{3} \backslash P_{3}\right)$ also cannot appear, because this would raise the constant energy density inside the black solid particle.

- Interaction $H_{1}^{+*} H_{02}$ finally reaches space case $8 C$, characterized by $\widetilde{P}_{1} \in \widetilde{p}_{02}$, with the analogous time case 4, characterized by $\widetilde{T}_{1} \in \tilde{\tau}_{02}$. This is the same interaction as in equation (34), for interaction $H_{1}^{+} * H_{04}$ generating a dot particle and a pellet particle. Compact spaces are not allowed to appear double and so interaction $H_{1}^{+} * H_{02}$ cannot generate anything. For interaction $H_{3}^{-*} H_{04}$, the same is valid.

All together, supposing that the final black solid particle is spherical, in the end we have one black solid particle $\sigma_{0204}\left(s^{02}\right)$ with black pellet particle $\pi_{0204}\left(p_{02} \cap p_{04}\right)$ upon its surface. Also upon its surface are charged dot particle $\delta_{104}^{+}\left(s^{1} \cap p_{04}\right)$ in $P_{1}$ with pellet particle $\pi_{104}\left(p_{1} \cap p_{04}\right)$ near, and charged dot particle $\pi_{104}\left(p_{1} \cap p_{04}\right)$ in $P_{3}$ with pellet particle $\pi_{302}\left(p_{3} \cap p_{02}\right)$ near (see Figure 15). No extended spaces appear anymore.

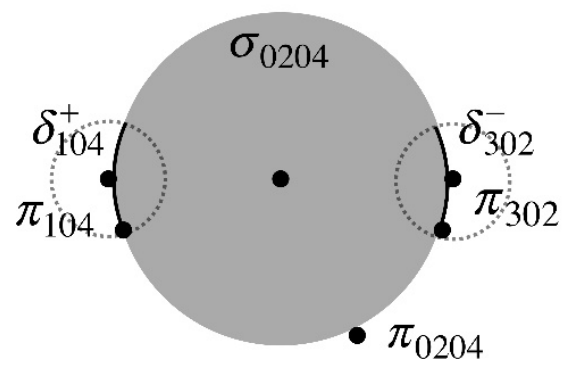

Figure 15. A spherical black solid particle with upon its surface a black pellet particle and two oppositely charged dot particles. No extended space appears 


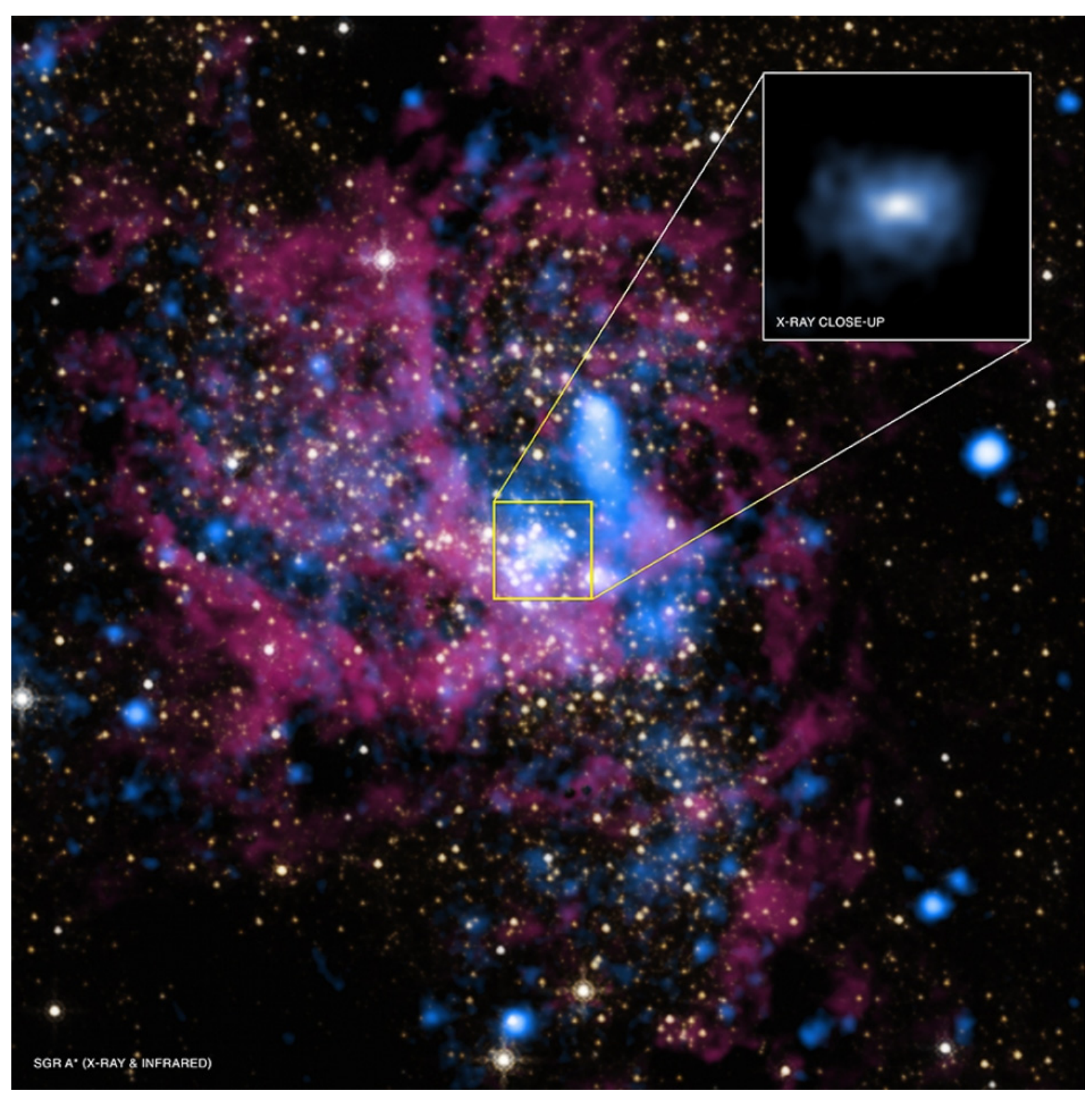

Figure 16. Sagittarius A* is the black hole at the center of the Milky Way galaxy.

X-ray: NASA/UMass/D.Wang et al., IR: NASA/STScI (Smith, 2017)

Summarizing, in the beginning we have four neutral H-units generating two black solid particles and a neutral macrospace. If inside the neutral macrospace marked H-units exist, then charged point particles and mixed macrospaces pop up around the two black solid particles. As soon as the black solid particles touch each other, two of the four neutral $\mathrm{H}$-units convert into marked $\mathrm{H}$-units and as a consequence, all previously generated items are annihilated. In the rearranged relationships, two smaller, oppositely charged solid particles pop up, a magnetic macrospace and a pellet particle.

We consider this as the birth of electromagnetism.

Then again a rearrangement occurs. The charged points of space are dragged away from their coinciding points, and the oppositely charged solid particles convert into oppositely charged dot particles, each with a pellet particle near. Shortly after that, one new black solid particle is generated, with a black pellet particle traveling over its surface. Also the two oppositely charged dot particles with their pellet particles finally are at the surface of the black solid particle. They might attract other charged objects towards the black solid particle, so these are not attracted by gravity, but by charged particles upon the surface.

In general, pairs of black solid particles will have the tendency, as soon as their major spaces start to overlap each other, to divide in those who are moving away from each other, and those who are approaching each other. 
This process is not disturbed by electromagnetic effects, so in this way a large number of black solid particles may gather in a relatively small area. If they interact two by two in the way as we explained in this chapter, then a huge number of neutral macrospaces might be generated. Because the energy density of neutral macrospaces is allowed to be added, and because they are astronomic large, this might result in an energy density above the allowed limit. Then the remaining interactions can generate only black solid particles and black pellet particles, without neutral macrospaces.

As soon as a pair of black solid particles, which is accompanied by a neutral macrospace, is close enough to touch each other's pellicles, it will be annihilated. Then a next pair of black solid particles gets the chance to generate a neutral macrospace. A huge amount of black solid particle pairs may be annihilated in a relatively short period of time, each pair apparently merging to one black solid particle, so a chain reaction occurs. All together an enormous amount of charged particles and magnetized macrospaces is to be expected and so a tremendous variety of electromagnetic activity around the interacting pairs of black holes, following up each other. This is in accordance with astronomic pictures (see Figure 16). Related to this chain of conversions from black solid particle pairs to single black solid particles, observations are expected to receive repeatedly extraordinary large signals. We suppose that these signals represent gravitational waves.

An oppositely charged pair of solid particles of type 2 will reach exactly the same six particles as described above, but the consideration for a large number is different. It is to be expected that developments, based upon electromagnetic interaction, will dominate spatial interactions and so this effect will occur as a secondary effect in the chain reaction.

\section{Energy Balance}

After having traced all physical items popping up during this complicated process, we will consider the energy balance.

In the beginning, the actual energy $E_{1}\left(H_{01} * H_{02} * H_{03} * H_{04}\right)$ of the total system can be written as:

$$
\begin{aligned}
& E_{1}\left(H_{01} * H_{02} * H_{03} * H_{04}\right)= \\
& E\left(H_{01} * H_{02}\right)+E\left(H_{03} * H_{04}\right)+E\left(H_{01} * H_{03}\right)+E\left(H_{01} * H_{04}\right)+E\left(H_{02} * H_{03}\right)+E\left(H_{02} * H_{04}\right),
\end{aligned}
$$

which is:

$$
E_{1}\left(H_{01} * H_{02} * H_{03} * H_{04}\right)=E\left(\sigma_{0102}+\pi_{0102}+\sigma_{0304}+\pi_{0304}+\Theta_{0103}+\Theta_{0104}+\Theta_{0203}+\Theta_{0204}\right) .
$$

At the end, the actual energy $E_{3}\left(H_{1}^{+*} H_{02} * H_{3}^{-*} H_{04}\right)$ of the total system can be written as:

$$
E_{2}\left(H_{1}^{+} * H_{02} * * H_{3}^{-} * H_{04}\right)=E\left(\sigma_{0204}+\pi_{104}+\delta_{104}^{+}+\pi_{0204}+\delta_{302}^{-}+\pi_{302}\right) .
$$

These expressions have to be equal, according to the law of conservation of actual energy, so:

$$
E_{2}=E_{1} \text {. }
$$

The actual energy of the involved black solid particles are equal, because they have the same size, so we drop $\sigma_{0102}$ and $\sigma_{0204}$. Supposing that the energy of the pellet particles upon the black hole is included in the energetic values of the belonging particles, we can drop them as well. The neutral macrospaces have the same energetic content and so we take four times one of them. Then equation (42) can be written as:

$$
E\left(\sigma_{0304}+4 \times \Theta_{0103}\right)=E\left(\delta_{104}^{+}+\delta_{302}^{-}+\Theta_{13}^{\mathbf{B}}\right) .
$$

This tells us that the energy of one black solid particle plus four neutral macrospaces is necessary to produce one pair of oppositely charged dot particles and one magnetic macrospace.

\section{Conclusion}

We started the description by considering four neutral H-units, two by two generating a black solid particle. Because gravitational waves are predicted by Einstein and he strongly believed that space and time have to be treated in a similar way, we used only analogous time and space cases. This turned out to be a useful help, because it reduced our choice from four to only one time case for each zipper; the resulting descriptions were compatible with experimental results. 
When two black solid particles, inside the addition of four identical neutral macrospaces, turn around each other while approaching, several particles and macrospaces appear and are annihilated. The two black solid particles do not merge as is suggested in the 33-seconds film 'Visualization of merging black holes and gravitational waves' (NASA, 2017), but are annihilated. Two neutral H-units are forced by the law of conservation of actual energy to create different identities and thus to generate oppositely charged solid particles, which is considered to be the birth of electromagnetism. This short period of reorganization is not observable, because it happens in the flying time in which no change can be described. After that, one new black solid particle is described, together with five much smaller particles, two of them being oppositely charged. The energy balance tells us that the space energy of one black solid particle plus four neutral macrospaces is necessary to generate one oppositely charged pair of particles and one magnetic macrospace.

This process of the apparent merging of black holes also may happen in larger black holes, each containing many black solid particles at a relatively short distance. This implies that the energy densities of the associated neutral macrospaces may add up to the maximum; after that, no further neutral macrospace can be generated. Only if the members of a pair of black solid particles, having a neutral macrospace, are close enough to touch each other's pellicles, their neutral macrospace will be annihilated. Then a subsequent pair of black solid particles gets the chance to generate one and so the process can continue, creating a chain reaction. Experimentally, the expectation is that extraordinarily large signals will be repeatedly received, related to this chain of annihilations of black solid particle pairs and subsequent generations of single black solid particles. We suppose that these signals represent gravitational waves. The variety of charged particles and magnetized spaces, popping up during this chain reaction, results in the tremendous electromagnetic activity which is observed around two large black holes.

\section{References}

Abbott, B. P., Abbott, R., Abbott, T. D., Abernathy, M. R., Acernese, F., Ackley, K., ... \& Adya, V. B. (2016). Observation of gravitational waves from a binary black hole merger. Physical review letters, 116(6), 061102.

Backerra, A. C. M. (2010). Uncertainty as a principle. Physics Essays, 23(3), 419-441.

Backerra, A. C. M. (2012). The unification of elementary particles. Physics Essays, 25(4), 601-619.

Backerra, A. C. M. (2014). The quantum-mechanical foundations of gravity. Physics Essays, 27(3), 380-397.

Backerra, A. C. M. (2016a). A bridge between quantum mechanics and astronomy. Applied Physics Research, $8(1), 16-40$.

Backerra, A. C. M. (2016b). The connection between gravity and electricity according to twin physics and a survey of the results so far, including neutron decay. Applied Physics Research, 8(6), 42-68.

Einstein, A. (1916). The foundation of the General Theory of Relativity. The collected papers of Albert Einstein, 8, Doc 30, 146-200, Trans. Retrieved from http://hermes.ffn.ub.es/luisnavarro/nuevo_maletin/Einstein GRelativity_1916.pdf

Einstein, A. (1996/ original 1936-1950). The theory of relativity (and other essays). Citadel Press Books, Carol Publishing Group edition.

Heisenberg, W. (1930/1949). The principles of the quantum theory. New York, NY: Dover publications.

Jammer, M. (1974). The Philosophy of Quantum Mechanics. New York, NY: John Wiley and Sons.

Kahn, P. J. (1967). Introduction to linear algebra. London: Harper \& Row, Ltd.

NASA, Kelly, B. J., Henze, C., \& Sandstrom, T. (2017, June 1). Visualization of merging black holes and gravitational waves. Retrieved from http://nasa.gov

Poincaré, H. (1905). Sur la dynamique de l' électron. Retrieved from http://www.academie-sciences.fr/pdf/ dossiers/Poincare/Poincare_pdf/Poincare_CR1905.pdf

Smith, H. R. (2017, March 8). What is a black hole? Retrieved from http://www.nasa.gov/audience/forstudents/ k-4 stories/nasa-knows/what-is-a-black-hole-k4.html 


\section{Appendix}

Set of attributes of one $\mathrm{H}$-unit in general:

$$
h_{i}=\left\{D_{i}, U^{i}, d_{i}, u^{i}\right\}
$$

Zipper in general:

$$
Z\left(H_{i} * H_{j}\right)=\left\{\begin{array}{l}
\left\{\left[D_{i} \propto D_{j}\right],\left[\left(D_{i} \bowtie u^{i}\right) \propto\left(D_{j} \bowtie u^{j}\right) \propto\left(D_{i} \bowtie u^{j}\right) \propto\left(D_{j} \bowtie u^{i}\right)\right]\right\} \\
\left\{\left[U^{i} \propto U^{j}\right],\left[\left(U^{i} \bowtie d_{i}\right) \propto\left(U^{j} \bowtie d_{j}\right) \propto\left(U^{i} \bowtie d_{j}\right) \propto\left(U^{j} \bowtie d_{i}\right)\right]\right\} \\
\left\{\left[D_{i} \propto U^{j}\right],\left[\left(D_{i} \bowtie u^{i}\right) \propto\left(U^{j} \bowtie d_{j}\right) \propto\left(D_{i} \bowtie d_{j}\right) \propto\left(U^{j} \bowtie u^{i}\right)\right]\right\} \\
\left\{\left[D_{j} \propto U^{i}\right],\left[\left(D_{j} \bowtie u^{j}\right) \propto\left(U^{i} \bowtie d_{i}\right) \propto\left(D_{j} \bowtie d_{i}\right) \propto\left(U^{i} \bowtie u^{j}\right)\right]\right\}
\end{array}\right\}
$$

Set of time attributes:

$$
h_{i}(\tilde{t})=\left\{\widetilde{T}_{i}, \widetilde{F}^{i} \backslash \widetilde{T}_{i}, \tilde{\tau}_{i}, \tilde{f}^{i}\right\}
$$

Set of space attributes:

$$
h_{i}(\tilde{\mathbf{x}})=\left\{\widetilde{P}_{i}, \tilde{S}^{i} \backslash \widetilde{P}_{i}, \tilde{p}_{i}, \tilde{s}^{i}\right\}
$$

Set of mark attributes:

$$
h_{i}(\tilde{q})=\left\{\left\{\widetilde{Q}_{i}, \widetilde{\mathbf{E}}_{i}\right\},\left\{\widetilde{Q}_{i} \times i, \widetilde{\mathbf{B}}^{i}\right\},\{1, \widetilde{\nabla}\},\{i, \partial / \partial \tilde{t}\}\right\}
$$

Time zipper in general:

$$
Z_{i j}(t)=\left\{\begin{array}{l}
\left\{\left[\widetilde{T}_{i} \cap \widetilde{T}_{j}\right],\left[\widetilde{f}^{i} \cap \widetilde{f}^{j} \cap\left(\widetilde{T}_{i \triangleright \triangleleft} \widetilde{f}^{j}\right) \cap\left(\widetilde{T}_{j \triangleright \triangleleft} \widetilde{f}^{i}\right)\right]\right\} \\
\left.\left\{\left[\widetilde{F}^{i} \backslash \widetilde{T}_{i} \cap \widetilde{F}^{j} \backslash \widetilde{T}_{j}\right],\left[\tilde{\tau}_{i} \cap \tilde{\tau}_{j} \cap\left(\widetilde{F}^{i} \backslash \widetilde{T}_{i \triangleright \triangleleft} \tilde{\tau}_{j}\right) \cap\left(\widetilde{F}^{j} \backslash \widetilde{T}_{j \triangleright \triangleleft} \tilde{\tau}_{i}\right)\right]\right\}\right\} \\
\left\{\left[\widetilde{T}_{i} \cap \widetilde{F}^{j} \backslash \widetilde{T}_{j}\right],\left[\widetilde{f}^{i} \cap \tilde{\tau}_{j} \cap\left(\widetilde{T}_{i \triangleright \triangleleft} \tilde{\tau}_{j}\right) \cap\left(\widetilde{F}^{j} \backslash \widetilde{T}_{j \triangleright \triangleleft} \widetilde{f}^{i}\right)\right]\right\} \\
\left\{\left[\widetilde{T}_{j} \cap \widetilde{F}^{i} \backslash \widetilde{T}_{i}\right],\left[\widetilde{f}^{j} \cap \tilde{\tau}_{i} \cap\left(\widetilde{T}_{j \triangleright \triangleleft} \tilde{\tau}_{i}\right) \cap\left(\widetilde{F}^{i} \backslash \widetilde{T}_{i \triangleright \triangleleft} \widetilde{f}^{j}\right)\right]\right\}
\end{array}\right\}
$$

Space zipper in general:

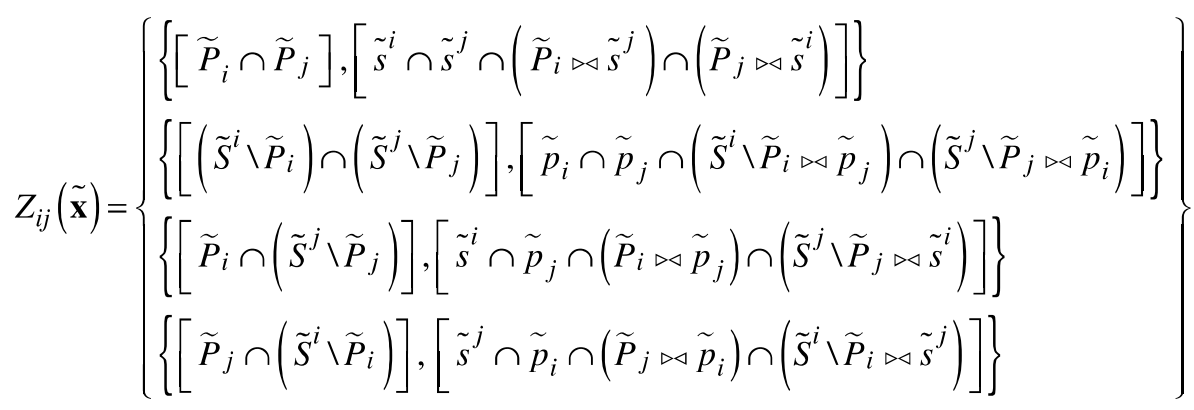

Mark zipper in general: 


$$
Z_{i j}(q)=\left\{\begin{array}{l}
\left\{\left[\left\{\tilde{Q}_{i} \propto \tilde{Q}_{j}, \tilde{\mathbf{E}}_{i} \propto \tilde{\mathbf{E}}_{j}\right\}\right],\left[\partial \tilde{\mathbf{E}}_{i} / \partial \tilde{t} \propto \partial \tilde{\mathbf{E}}_{j} / \partial \tilde{t}\right]\right\} \\
\left\{\left\{\left[\left(\tilde{Q}_{i} \times i\right) \propto\left(\tilde{Q}_{j} \times i\right)\right],\left[\tilde{\mathbf{B}}^{i} \propto \tilde{\mathbf{B}}^{j}\right]\right\},\left[\tilde{\nabla} \times \tilde{\mathbf{B}}^{i} \propto \tilde{\nabla} \times \tilde{\mathbf{B}}^{j}\right]\right\} \\
\left.\left\{\left[\left\{\tilde{Q}_{i} \propto\left(\tilde{Q}_{j} \times i\right), \tilde{\mathbf{E}}_{i} \propto \tilde{\mathbf{B}}^{j}\right\}\right],\left[\partial \tilde{\mathbf{E}}_{i} / \partial \tilde{t} \propto \tilde{\nabla} \times \tilde{\mathbf{B}}^{j} \propto \tilde{\nabla} \times \tilde{\mathbf{E}}_{i} \propto \partial \tilde{\mathbf{B}}^{j} / \partial \tilde{t}\right]\right\}\right\} \\
\left.\left\{\left[\left\{\tilde{Q}_{j} \propto\left(\tilde{Q}_{i} \times i\right), \tilde{\mathbf{E}}_{j} \propto \tilde{\mathbf{B}}^{i}\right\}\right],\left[\partial \tilde{\mathbf{E}}_{j} / \partial \tilde{t} \propto \tilde{\nabla} \times \tilde{\mathbf{B}}^{i} \propto \tilde{\nabla} \times \tilde{\mathbf{E}}_{j} \propto \partial \tilde{\mathbf{B}}^{i} / \partial \tilde{t}\right]\right\}\right\}
\end{array}\right\}
$$

\section{Time cases:}

The time zipper of $H_{i} * H_{j}$ and the time-mirrored interaction $H_{j}{ }^{*} H_{i}$ are in general not equal; then both are given.

Time case 1: $\widetilde{T}_{i} \cap \widetilde{T}_{j}=\widetilde{T}_{i}$.

$$
Z_{i j}(t)=\left\{\left\{T_{i}, f^{i}\right\},\left\{F^{i} \backslash T_{i}, d t\right\}, \varnothing, \varnothing\right\}
$$

Time case 2: $\widetilde{T}_{i} \cap \widetilde{T}_{j}=\varnothing$ and $\tilde{\tau}_{i} \cap \tilde{\tau}_{j} \neq \varnothing$. For $H_{i}^{*} H_{j}$ is:

$$
Z_{i j}(t)=\left\{\varnothing,\left\{F^{i} \backslash T_{i} \cap F^{j}, d t^{2}\right\},\left\{T_{i}, \varnothing\right\}, \varnothing\right\} .
$$

For $H_{j}{ }^{*} H_{i}$ is:

$$
Z_{j i}(t)=\left\{\varnothing,\left\{F^{j} \backslash T_{j} \cap F^{i}, d t^{2}\right\}, \varnothing,\left\{T_{j}, \varnothing\right\}\right\} .
$$

Time case 3 in general: $\tilde{\tau}_{i} \cap \tilde{\tau}_{j}=\varnothing$.

For $H_{i}^{*} H_{j}$ is:

$$
Z_{i j}(t)=\left\{\varnothing,\left\{F^{i} \backslash T_{i} \cap F^{j}, \varnothing\right\},\left\{T_{i}, \varnothing\right\}, \varnothing\right\} .
$$

For $H_{j}^{*} H_{i}$ is:

$$
Z_{j i}(t)=\left\{\varnothing,\left\{F^{j} \backslash T_{j} \cap F^{i}, \varnothing\right\}, \varnothing,\left\{T_{j}, \varnothing\right\}\right\} .
$$

There are two relative positions possible to achieve one of these zippers:

Time case 3A: $\tilde{\tau}_{i} \cap \tilde{\tau}_{j}=\varnothing$ and $\widetilde{T}_{i} \in \widetilde{f}^{j}$ (or if mirrored: $\widetilde{T}_{j} \in \widetilde{f}^{i}$ ).

Time case 3B: $\tilde{\tau}_{i} \cap \tilde{\tau}_{j}=\varnothing$ and $\widetilde{F}^{i} \cap \tilde{\tau}_{j}=\varnothing$ (or if mirrored: $\widetilde{F}^{j} \cap \tilde{\tau}_{i}=\varnothing$ ). 
Time case 4: $\widetilde{T}_{i} \in \tilde{\tau}_{j}$ or $\widetilde{T}_{j} \in \tilde{\tau}_{i}$.

For $H_{i}^{*} H_{j}$ is:

$$
Z_{i j}(t)=\left\{\varnothing,\left\{F^{i} \backslash T_{i} \cap F^{j}, \varnothing\right\},\left\{T_{i}, f^{i} \cap d t\right\}, \varnothing\right\} .
$$

For $H_{j} * H_{i}$ is:

$$
Z_{j i}(t)=\left\{\varnothing,\left\{F^{j} \backslash T_{j} \cap F^{i}, \varnothing\right\}, \varnothing,\left\{T_{j}, f^{j} \cap d t\right\}\right\} .
$$

Space cases for equally sized H-units (so for $H_{i} * H_{j}$ or $H_{0 i}{ }^{*} H_{0 j}$ ): The space zipper of $H_{i}{ }^{*} H_{j}$ is equal to the space zipper of time-mirrored interaction $H_{j} * H_{i}$, so the indices do not have to be interchanged.

Space case 1: $\widetilde{P}_{i} \cap \widetilde{P}_{j}=\widetilde{P}_{i}$.

$$
Z_{i j}(\mathbf{x})=\left\{\left\{P_{i}, \theta\left(s^{i}\right)\right\},\left\{\Theta\left(S^{i} \backslash P_{i}\right), \circ\left(p_{i}\right)\right\}, \varnothing, \varnothing\right\}
$$

Space case 2: $\widetilde{P}_{i} \cap \widetilde{P}_{j}=\varnothing, \widetilde{P}_{i} \in \tilde{S}^{j}$ and so $\widetilde{P}_{j} \in \tilde{s}^{i}$.

$$
Z_{i j}(\mathbf{x})=\left\{\left\{\varnothing, \theta\left(s^{i} \cap s^{j}\right)\right\},\left\{\Theta\left(S^{i} \backslash P_{i} \cap S^{j} \backslash P_{j}\right), \circ\left(p_{i} \cap p_{j}\right)\right\},\left\{P_{i}, \varnothing\right\},\left\{P_{j}, \varnothing\right\}\right\}
$$

Space case 3: $\widetilde{P}_{i} \in \widetilde{p}_{j}$ and so $\widetilde{P}_{j} \in \widetilde{p}_{i}$.

$$
Z_{i j}(\mathbf{x})=\left\{\varnothing,\left\{\Theta\left(S^{i} \backslash P_{i} \cap S^{j} \backslash P_{j}\right), \circ\left(p_{i} \cap p_{j}\right)\right\},\left\{P_{i}, \odot\left(s^{i} \cap p_{j}\right)\right\},\left\{P_{j}, \odot\left(s^{j} \cap p_{i}\right)\right\}\right\}
$$

Space case 4: $\tilde{s}^{i} \cap \tilde{s}^{j} \neq \varnothing, \quad \widetilde{P}_{i} \notin \tilde{s}^{i} \cap \tilde{s}^{j}$ and so $\widetilde{P}_{j} \notin \tilde{s}^{i} \cap \tilde{s}^{j}$.

$$
Z_{i j}(\mathbf{x})=\left\{\varnothing,\left\{\Theta\left(S^{i} \backslash P_{i} \cap S^{j} \backslash P_{j}\right), \circ\left(p_{i} \cap p_{j}\right)\right\},\left\{P_{i}, \varnothing\right\},\left\{P_{j}, \varnothing\right\}\right\}
$$

Space case 5: $\tilde{p}_{i} \cap \tilde{p}_{j}=\tilde{p}_{i j}$.

$$
Z_{i j}(\mathbf{x})=\left\{\varnothing,\left\{\Theta\left(S^{i} \backslash P_{i} \cap S^{j} \backslash P_{j}\right), \circ\left(p_{i j}\right)\right\},\left\{P_{i}, \varnothing\right\},\left\{P_{j}, \varnothing\right\}\right\}
$$

Space case 6: $\tilde{p}_{i} \cap \tilde{p}_{j}=\varnothing, \quad \widetilde{P}_{i} \in \tilde{S}^{j}$ and so $\widetilde{P}_{j} \in \tilde{S}^{i}$

$$
Z_{i j}(\mathbf{x})=\left\{\varnothing,\left\{\Theta\left(S^{i} \backslash P_{i} \cap S^{j} \backslash P_{j}\right), \varnothing\right\},\left\{P_{i}, \varnothing\right\},\left\{P_{j}, \varnothing\right\}\right\}
$$

Space case 7: $\tilde{S}^{i} \cap \tilde{S}^{j} \neq \varnothing, \widetilde{P}_{i} \notin \tilde{S}^{j}$ and so $\widetilde{P}_{j} \notin \tilde{S}^{i}$.

$$
Z_{i j}(\mathbf{x})=\left\{\varnothing,\left\{\Theta\left(S^{i} \cap S^{j}\right), \varnothing\right\}, \varnothing, \varnothing\right\}
$$




\section{Analogous space and time cases:}

space case 1 and time case 1

space case 2 and time case 1

space case 2 and time case $3 \mathrm{~A}$

space case 3 and time case 4

space case 4 or 5 and time case 2

space case 6 or 7 and time case 3B

\section{Space cases for different sized H-units, only as far as used:}

The space zipper of $H_{i} * H_{0}$ is equal to the space zipper of time-mirrored interaction $H_{0} * H_{i}$, so the indices do not have to be interchanged for the mirrored interaction.

Space case 7B: $\tilde{p}_{i} \cap \tilde{p}_{0}=\varnothing, \quad \widetilde{P}_{i} \in \tilde{S}^{0}$ and $\widetilde{P}_{0} \notin \tilde{S}^{i}$

$$
Z_{i 0}(\mathbf{x})=\left\{\varnothing,\left\{\Theta\left(S^{i} \backslash P_{i} \cap S^{0} \backslash P_{0}\right), \varnothing\right\},\left\{P_{i}, \varnothing\right\}, \varnothing\right\}
$$

Space case 7C: $\tilde{p}_{i} \cap \tilde{p}_{0}=\varnothing, \widetilde{P}_{i} \in \tilde{S}^{0}$ and $\widetilde{P}_{0} \in \tilde{S}^{i}$.

$$
Z_{i 0}(\mathbf{x})=\left\{\varnothing,\left\{\Theta\left(S^{i} \backslash P_{i} \cap S^{0} \backslash P_{0}\right), \varnothing\right\},\left\{P_{i}, \varnothing\right\},\left\{P_{0}, \varnothing\right\}\right\}
$$

Space case 8C: $\widetilde{P}_{i} \in \widetilde{p}_{0}$.

$$
Z_{i 0}(\mathbf{x})=\left\{\varnothing,\left\{\Theta\left(S^{i} \backslash P_{i}\right), \circ\left(p_{i} \cap p_{0}\right)\right\},\left\{P_{i}, \odot\left(s^{i} \cap p_{0}\right)\right\},\left\{P_{0}, \varnothing\right\}\right\}
$$

Space case 9D: $\widetilde{P}_{i} \cap \widetilde{P}_{0}=\widetilde{P}_{i}$.

$$
Z_{i 0}(\mathbf{x})=\left\{\left\{P_{i}, s^{i}\right\},\left\{\Theta\left(S^{i} \backslash P_{i}\right), \varnothing\right\},\left\{P_{i}, \varnothing\right\},\left\{P_{0}, \varnothing\right\}\right\} .
$$

\section{Analogous space and time cases:}

space case $7 \mathrm{~B}$ and time case $3 \mathrm{~B}$

space case $8 \mathrm{C}$ and time case 4

space case 9D and time case 1

\section{Copyrights}

Copyright for this article is retained by the author(s), with first publication rights granted to the journal.

This is an open-access article distributed under the terms and conditions of the Creative Commons Attribution license (http://creativecommons.org/licenses/by/4.0/). 